\title{
Diagnosis and key of the main families and species of South American Coleoptera of forensic importance ${ }^{1}$
}

\author{
Lúcia M. Almeida² \& Kleber M. Mise ${ }^{3}$
}

\author{
${ }^{1}$ Contribution number 1768 of the Department of Zoology, Universidade Federal do Paraná, Brazil. \\ ${ }^{2}$ Departamento de Zoologia, Universidade Federal do Paraná, Caixa Postal 19020, 81581-980 Curitiba-PR, Brasil. Fellowship CNPq. \\ lalmeida@ufpr.br \\ ${ }^{3}$ Fellowship CAPES. klebermise@yahoo.com.br
}

\begin{abstract}
Diagnosis and key of the main families and species of South American Coleoptera of forensic importance. The objective of this paper is to provide diagnosis and keys of the families and species, with illustrations of the main groups. A table of all related species recorded from South America is presented, including the substrate in which they were collected and their geographical distribution. The list comprises 221 species included in 15 families, of which $70 \%$ of the species are from Brazil. Scarabaeidae is the most diverse family with 121 species, followed by Staphylinidae with 68. Also we provide one database of Coleoptera species associated with carcasses in South America.
\end{abstract}

KEYWORDS. Forensic entomology; necrophilous beetles; Neotropical region; taxonomy.

RESUMO. Diagnose e chave de identificação para as principais famílias e espécies de Coleoptera de importância forense da América do Sul. O objetivo deste trabalho é apresentar diagnoses e chaves de identificação das principais famílias e espécies de importância forense, com ilustrações dos principais grupos. É apresentada uma tabela de todas as espécies de ocorrência na América do Sul, incluindo o substrato nas quais foram coletadas e sua distribuição geográfica. A lista compreende 221 espécies incluídas em 15 famílias, das quais pelo menos $70 \%$ das espécies são distribuídas no Brasil. Scarabaeidae é a família com maior diversidade com 121 espécies, seguida por Staphylinidae com 68. Também é fornecida uma base de dados para as espécies de Coleoptera associadas a carcaças na América do Sul.

PALAVRAS-CHAVE. Besouros necrófilos; entomologia forense; neotropical; taxonomia.

Coleoptera is the second largest order of forensic interest, with several necrophagous representatives, most being predators but their feeding habit may change between larval stages and adulthood. The species of Coleoptera increase in number both of individuals and species during advanced stages of decomposition in open environment and are absent or less represented indoors (Goff 1991). Beetles are encountered in great numbers during the faunal succession process, moreover their biological traits may be used to estimate the post mortem interval.

According to Smith (1986) the families of Coleoptera of forensic interest are: Carabidae, Hydrophilidae, Silphidae, Leiodidae, Staphylinidae, Histeridae, Cleridae, Anthicidae, Dermestidae, Nitidulidae, Rhizophagidae, Ptinidae, Tenebrionidae, Scarabaeidae, Geotrupidae and Trogidae.

In Brazil one of the most comprehensive studies of this fauna was done by Luederwaldt (1911), who found about 62 species related to carcasses. Subsequently papers were mainly focused on Diptera, but some of them reported Coleoptera species.

Some authors aimed on specific families contributing with taxonomic or survey studies of necrophilous beetles. Pessôa \& Lane (1941) did studies on Scarabaeinae fauna of legal medicine interest, found in São Paulo and neighborhood (southeast of Brazil) comprising 113 species of 26 genera.
The fauna of Staphylinidae was studied by JimenezSanchez et al. (2000) on Nanchititla, Mexico. The 50 species in their study were collected with traps using squid as bait.

Scampini et al. (2002) found six species of Carabidae from Buenos Aires (Santa Catalina), Argentina, using pig carcasses and pit-fall traps during three months.

Souza \& Linhares (1997) studied Diptera and Coleoptera collected in pig carcass and reported 13 species in five families, of which Staphylinidae had greater number of species, only two being identified to the species level.

A study conducted by Mise et al. (2007) focusing on Coleoptera took place in Curitiba for one year using pig carcass with Shannon modified, pit-fall traps and active sampling. There were found 4,360 beetles belonging to 112 species of 26 families, 12 of them were considered of forensic potential.

In South America and in Brazil, the majority of studies focuses mainly on Diptera, one of the reasons is due to difficulties in identifying the species of Coleoptera. There are no published records of Coleoptera of forensic importance in South America in a checklist and this data will be useful to start a database of the fauna of Coleoptera associated with different kinds of carcasses.

Due to the importance of the beetles to forensic sciences and the few studies in the Coleoptera fauna of South America, the purpose of this paper is to provide data about the families 
and species, with brief descriptions, identification keys and illustrations for the main groups. Also when available the alimentary habits of the species had been added.

\section{MATERIALAND METHODS}

Taxa were arranged in alphabetical order at family, genera and species level, when possible, followed by the substratum, geographical distribution and references. White (1983), Booth et al. (1990), Kingsolver (1991), Borror et al. (1992), Costa (2000), Newton et al. (2005) and Costa et al. (2006) were used for the families descriptions, their ecological data and number of genera and species.

The list of the important groups of Coleoptera show different species found on different animal carcasses across the Neotropical region. Many species were reported only once because there is a huge difference among regions. This could be due to the endemism of certain species, outlining the importance of regional surveys, which could indicate the species of Coleoptera of potential forensic importance.

The keys from the most general to the most specific, deal with beetles associated with decomposing animal carcasses. Accidental families, genera and species are not included because the lack of specimens or literature.

\section{RESULTS}

The list of the main Coleoptera species of forensic importance has 221 species included in 15 families; however this is a conservative estimate, considering that most of the specimens cited in papers are not identified at species level (Table I). At least $70 \%$ of the species occurs in Brazil, being Scarabaeidae the most diverse family with 122 species, followed by Staphylinidae with 69. Also we provide a diagnosis and keys of the families and species, with illustrations to enable the identification of the main groups.
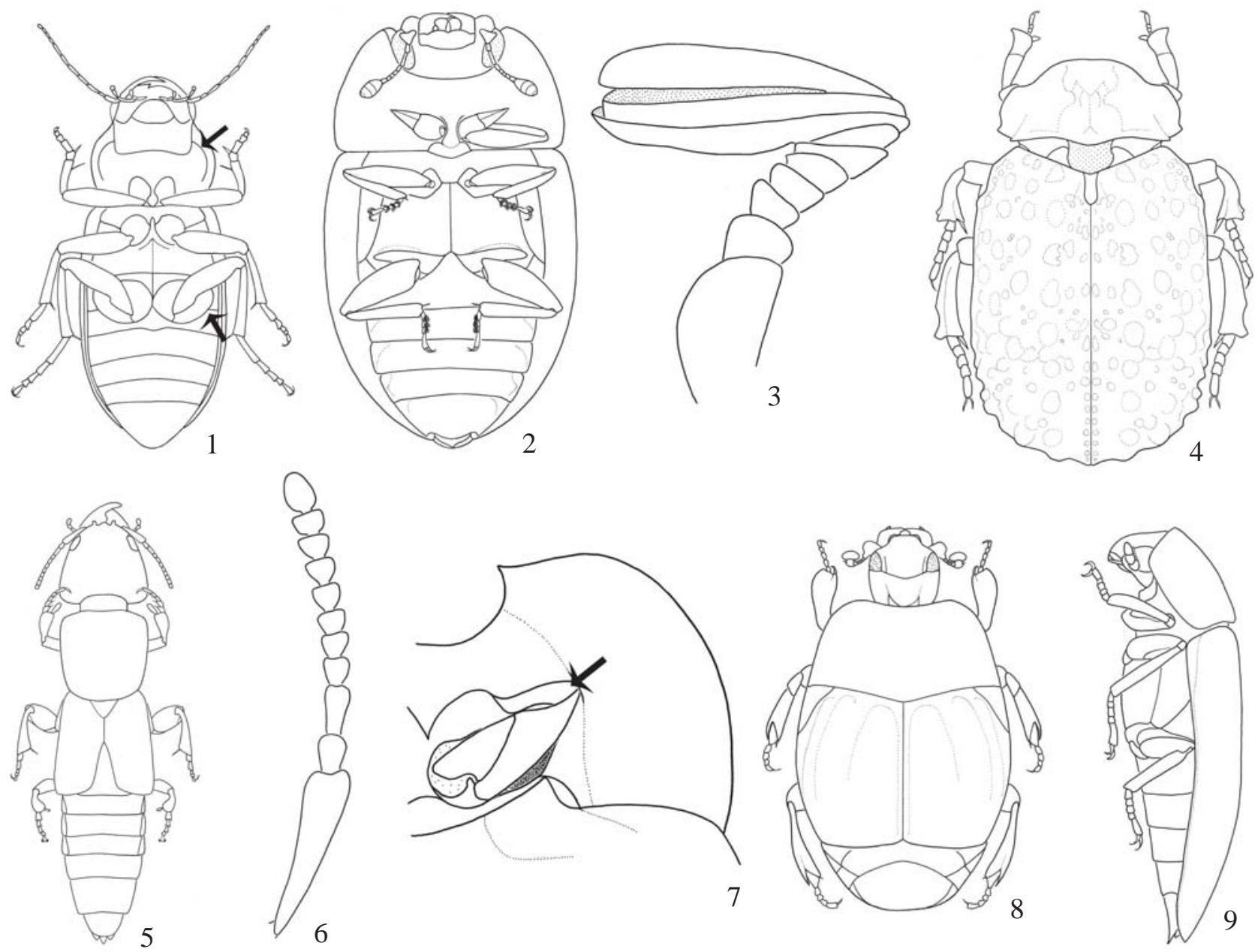

Figs. 1-9. Ventral view: 1, Adephaga (notopleural suture; first abdominal sternite divided by metacoxal cavities); 2, Polyphaga; 3, lamellated antennae: Scarabaeidae; dorsal view: 4, Polynoncus sp. (Trogidae); 5, Eulissus chalybaeus (Staphylinidae); 6, antennae: Staphylinidae; 7 , prothorax ventral view: Oxelytrum discicolle (Silphidae) (exposed trochantin); 8, dorsal view: Histeridae; 9, lateral view: Dermestes maculatus (Dermestidae). 

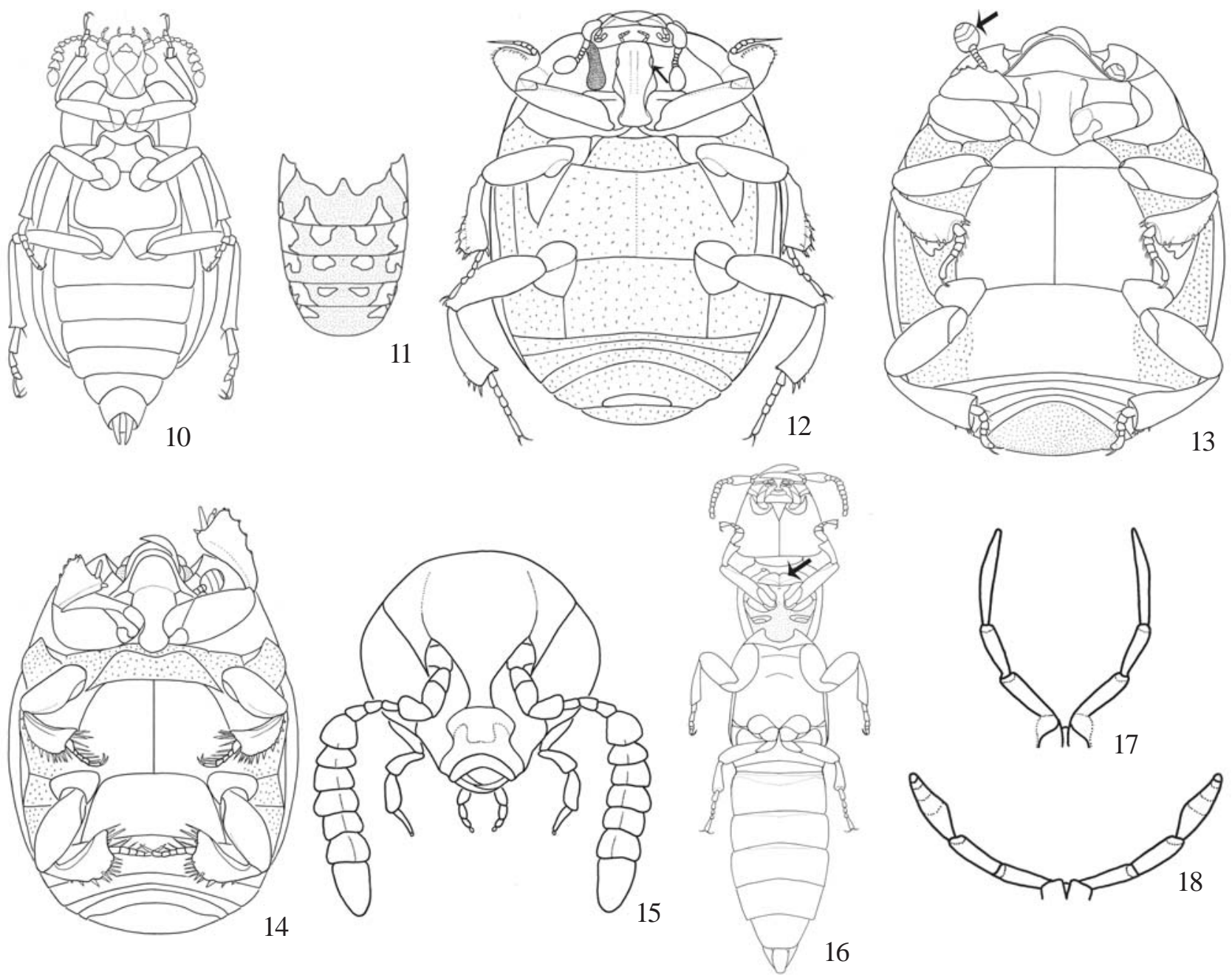

Figs. 10-18. Ventral view: 10, Oxelytrum discicolle (Silphidae); 11, abdomen: Dermestes ater (Dermestidae); 12, Euspilotus nigrita (Histeridae) (preapical fovea); 13, Omalodes sp. (Histeridae) (V-shaped antennal sutures); 14, Hister sp. (Histeridae); 15, frontal view of head: Aleochara sp. (Staphylinidae); 16, ventral view: Eulissus chalybaeus (Staphylinidae) (sclerotized plate in front of prosternum); maxillary palpi: 17, Belonuchus sp.; 18, Philonthus sp. (Staphylinidae).

Key of South American Coleoptera of forensic importance 1. Notopleural suture distinct; metatrochanter very large; first visible abdominal sternite divided by metacoxae (Fig. 1) (Suborder Adephaga) Carabidae Without notopleural suture; first visible abdominal sternite entire (Fig. 2) (Suborder Polyphaga) 2

2. Antennae lamellated (Fig. 3)

Antennae not lamellated ... 3

3. Clypeus large covering labrum in dorsal view; mandible usually hidden from above; abdomen with 5 or 6 visible segments Scarabaeidae

Head bent down almost hypognathous; labrum distinct and bent down; mandible proeminent not covered by clypeus; elytra often with tubercles (Fig. 4); abdomen with 5 visible segments Trogidae
4. Head prognathous; elytra usually very short and truncate, exposing more than three abdominal tergites (Fig. 5); antennae filiform or moniliform, occasionally with weak club (Fig. 6); tarsal formula variable, 3-3-3 to 5-5-5; abdomen usually capable of being flexed ... Staphylinidae

Without the above combination of characters 5

5. Antennae with a compact club; elytra often short and truncate exposing abdominal tergites or entire ... 6

Without the above combination of characters 7

6. Procoxae transverse with exposed trochantin (Fig. 7); tarsal formula often 5-5-5 with $4^{\text {th }}$ reduced Nitidulidae

Procoxae transverse without exposed trochantin; elytra often short and truncate exposing pygidium and propygidium (Fig. 8); tibiae flattened with spines or teeth, tarsal formula 5-5-5, rarely 5-5-4 Histeridae 

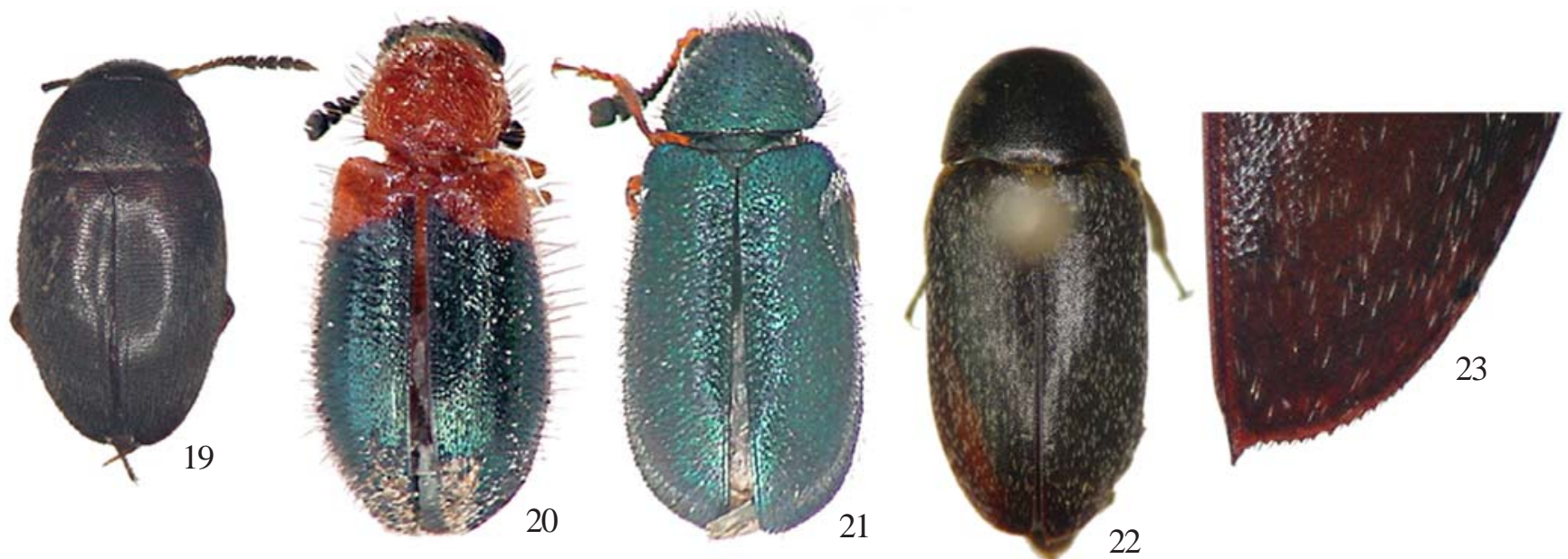

Figs. 19-23. Habitus: 19, Dissochaetus murray (Leiodidae); 20, Necrobia ruficollis; 21, Necrobia rufipes (Cleridae); 22, Dermestes maculatus; 23, elytral apex: Dermestes maculatus (Dermestidae).

7. Body usually oval in shape; sometimes parallel sided; antennae short with a four segmented club, basal segment shiny, the apical three segments pubescent; maxillary palpi long often longer than antennae; procoxae conical; tibiae often spinose with two usually large spurs; tarsi usually 5-5-5; abdomen with 5 visible sternites

Hydrophilidae

Without the above combination of characters 8

8. Tarsal formula 5-5-4 Tenebrionidae

Tarsal formula 5-5-5 9

9. Head flattened, inserted in broad imargination of pronotum; antennae 10-11 segmented usually capitate with a loose club but may be compact, club 3-5 segmented; abdomen with 5 visible sternites the sixth often partially visible Leiodidae

Body castaneus covered with decumbent hairs; antennae with a loose club, the eighth segment reduced and the eleventh pointed; approximately $2,33 \mathrm{~mm}$ (Fig. 19) Dissochaetus murray Reitter, 1884 Without the above combination of characters .. 10

10.Head hypognathous (Fig. 9) 11

Head not as above; antennae sub-clubbed or clubbed with 10 or 11 segments (Fig. 10), segments 9-11 with dense pubescence; trochantin exposed (Fig. 7); elytra often with longitudinal striae, color black, testaceous, or black with orange or yellow markings, sometimes with pronotum tomentose, parcial or totally colored of yellow or reddish

Silphidae

11.Metacoxae often excavated for reception of metafemora; frons often with median ocellus; antennae short usually clubbed often received into grooves on underside of prothorax; five visible sternites Dermestidae
Body elongated, covered with bristly hairs; frons oblique and eyes large; antennae not received into grooves on underside of prothorax; pronotum narrower than elytra, often nearly cylindrical; procoxae usually conical; tarsal formula 5-5-5 or sometimes pseudotetramerous .. Cleridae
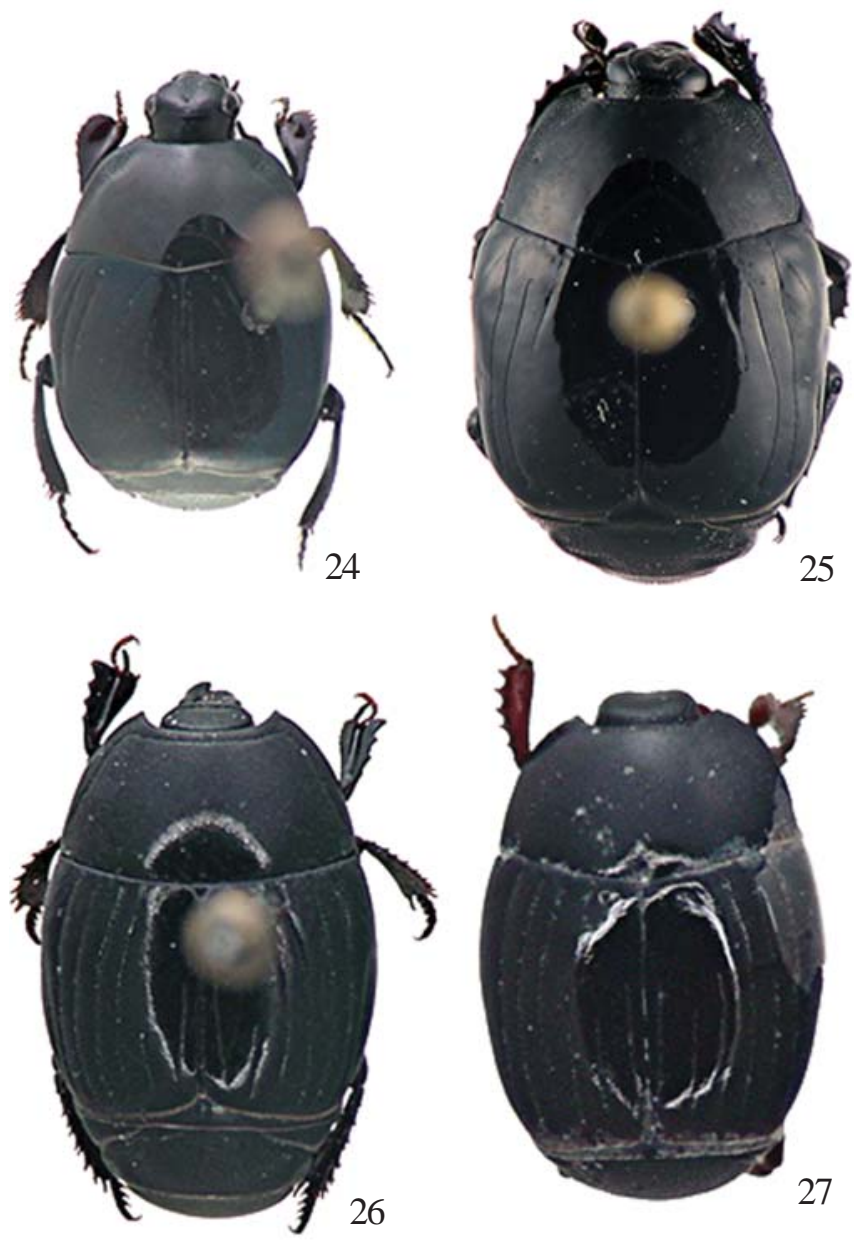

Figs. 24-27. Habitus of Histeridae. 24, Euspilotus nigrita; 25, Omalodes bifoveolatus; 26, Hister sp.; 27, Phelister sp.. 

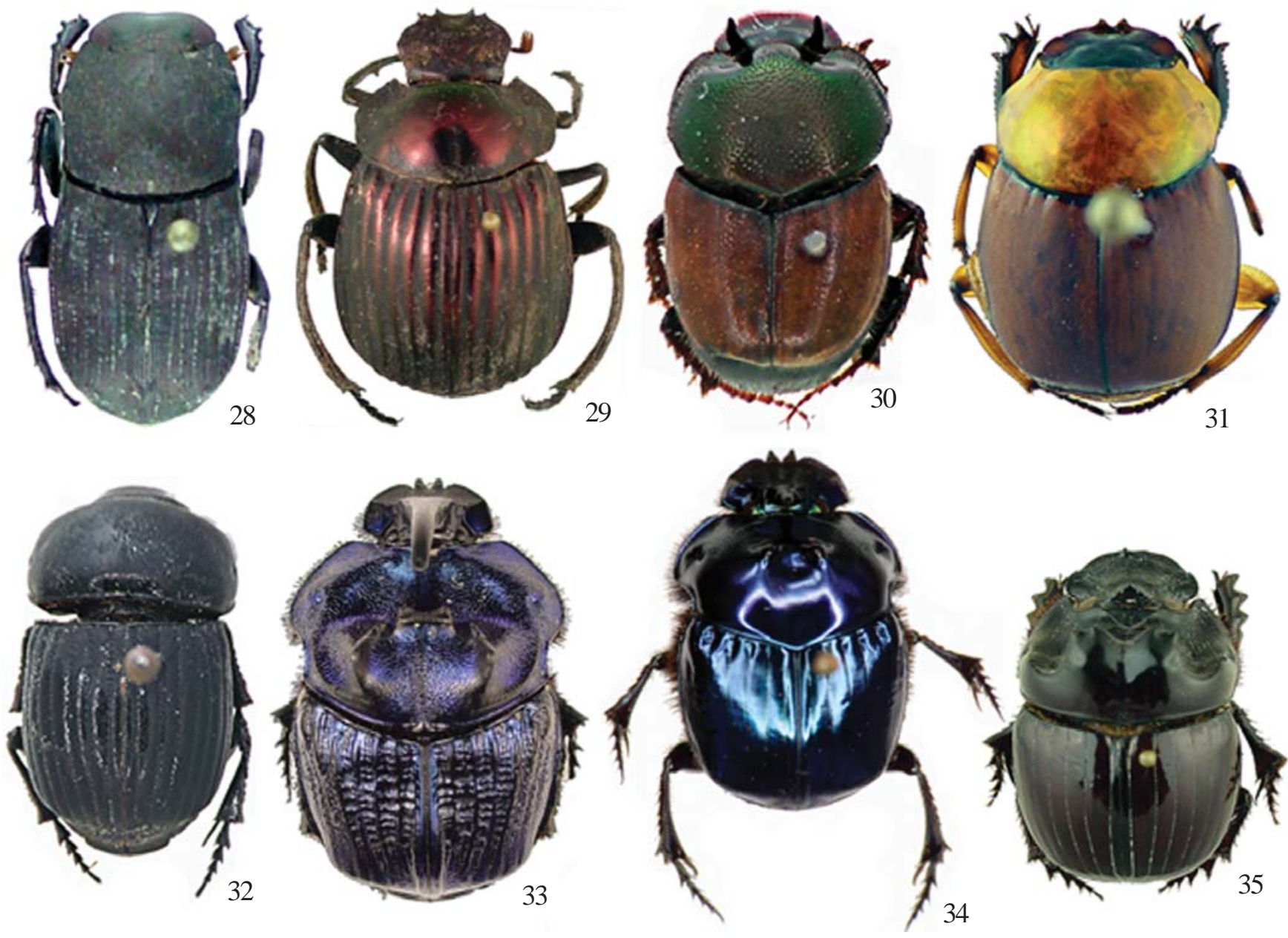

Figs. 28-35. Habitus of Scarabaeidae. 28, Eurysternus sp.; 29, Deltochilum icarus; 30, Onthophagus buculus; 31, Canthon triangularis; 32, Ontherus sp.; 33, Coprophanaeus lancifer; 34, Coprophanaeus saphirinus; 35, Dichotomius boreus.

Key to species of Cleridae

1. Head, pronotum and elytra base reddish brown and the rest of the elytra metallic blue; approximately 4,77 mm (Fig. 20) Necrobia ruficollis (Fabricius, 1775) Body metallic blue (Fig. 21); approximately 5,17 mm .... Necrobia rufipes (De Geer, 1775)

Key to species of Dermestidae

1. Elytra apex serrate with a small terminal spine (Figs. $22 \mathrm{e}$ 23); approximately $9,16 \mathrm{~mm}$

Dermestes maculatus (De Geer, 1774)

Elytra apex entire lacking serration and spines

... 2

2. Abdominal venter without pattern; lateral sulcus of abdominal sternum I closely parallel to lateral margin; approximately $8,5 \mathrm{~mm}$

Dermestes peruvianus Laporte, 1840

Abdominal venter patterned (Fig. 11); lateral sulcus of abdominal sternum I not closely parallel to lateral margin; approximately $8 \mathrm{~mm}$....... Dermestes ater (De Geer, 1774)
Key to species of Histeridae

1. Small sized beetles $(<0,3 \mathrm{~mm})$; antennal insertions usually exposed; elytra at most with vague impressions; scutellum hidden; hind tarsi with four segments .. Aeletes

Without the above combination of characters; large sized beetles $(>0,3 \mathrm{~mm})$

2. Prosternum with an antennal cavity for reception of antennae

Prosternum not as above 4

3. Prosternal process with a preapical fovea (Fig. 12) ....... Euspilotus

Lateral lobe of the abdominal $8^{\text {th }}$ tergite with rounded apex; pygidial line rounded at apex (sometimes weakly sinuated or interrupted); size approximately 7,66 mm (Fig. 24) . Euspilotus nigrita (Blanchard, 1842)

Prosternal process without a preapical fovea ....... Saprinus

4. Antennal club with two oblique sutures, V-shaped (Figs. 13 e 25) Omalodes 

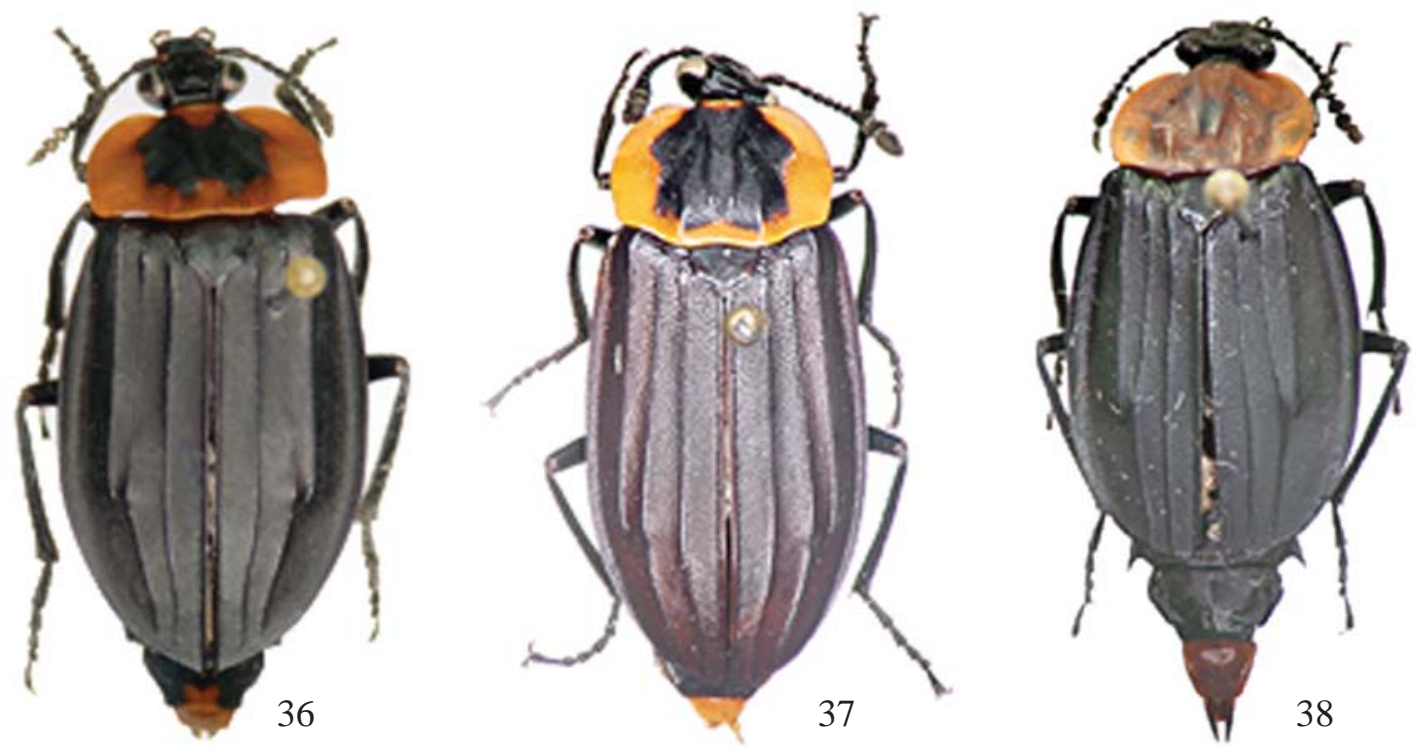

Figs. 36-38. Habitus of Silphidae. 36, Oxelytrum discicolle; 37, Oxelytrum erythrurum; 38, Oxelytrum cayennense.

Pronotum with two fovea on the lateral sides; size approximately $11 \mathrm{~mm}$ (Fig. 25)

Omalodes bifoveolatus (Marseul, 1853)

Antennal club not as above 4

5. Antennal club with two annuli (Fig. 14); frontal suture, if present, distant from antennal base (Fig. 26) ....... Hister

Antennal club with only a straight, transverse subapical annulus of short setae; frontal striae reaching the antennal base, if present (Fig. 27) Phelister

Key to species of Scarabaeidae

1. Mid coxae with external lateral border parallel to the body axis and located in the lateral of the metasternum in the limit of the lateral border of the body (Fig. 28)

Eurysternus

Without the above combination of characters ... 2

2. Elytral apex with carinae or distinct tubercles between the interstriae

Deltochilum

Clypeus with four teeth; elytrae with numerous striae; metallic colored; size approximately 26 mm (Fig. 29)

Deltochilum icarus (Olivier, 1789)

Without the above combination of characters 3

3. Body length less than $12 \mathrm{~mm}$; elytra and pronotum hairy; first tarsal segment of posterior leg almost as longer as the others together (Fig. 30) Onthophagus

Without the above combination of characters 4

4. Pigidium flattened, with dull shine Canthon Pronotum shining yellow colored; elytrae brownish-yellow and opacous; size approximately $11 \mathrm{~mm}$ (Fig. 31) ........ . Canthon triangularis (Drury, 1770)

Without the above combination of characters
5. Body oval elongated; colour usually black, sometimes green or blue; abdominal sternites fused and very short (Fig. 32)

Ontherus

Without the above combination of characters 6

6. Antennal club with segments wide and flattened; anterior margin of clypeus with three emarginations, with two proeminent teeth and two lateral round angles; first segment of mid and hind tarsus elongated, longer than wide

Coprophanaeus

Metallic bluish colored with integument hardly puncturate; size approximately $37 \mathrm{~mm}$ (Fig. 33)

Coprophaneus lancifer (Linnaeus, 1767)

Metallic bluish colored with integument smooth; size approximately $19 \mathrm{~mm}$ (Fig. 34)

Coprophaneus saphirinus (Sturm, 1826)

7. Antennal club with segments elongated and thin; anterior margin of clypeus without emargination, with two short teeth Dichotomius

Protibiae with a slender spine in the apice; size approximately $22 \mathrm{~mm}$ (Fig. 35) . Dichotomius boreus (Olivier, 1789)

Key to species of Silphidae

1. With a tooth in the humeral region of the elytra; size approximately $17 \mathrm{~mm}$ (Fig. 36)

Oxelytrum discicolle (Brullé, 1840)

Without a tooth or only with a small protuberance in the humeral region of the elytra; size approximately $16 \mathrm{~mm}$...

2

2. Pronotum with a maculae quadrangular-shaped, occupying most of the pronotal disk (Fig. 37) Oxelytrum erythrurum (Blanchard, 1840) 

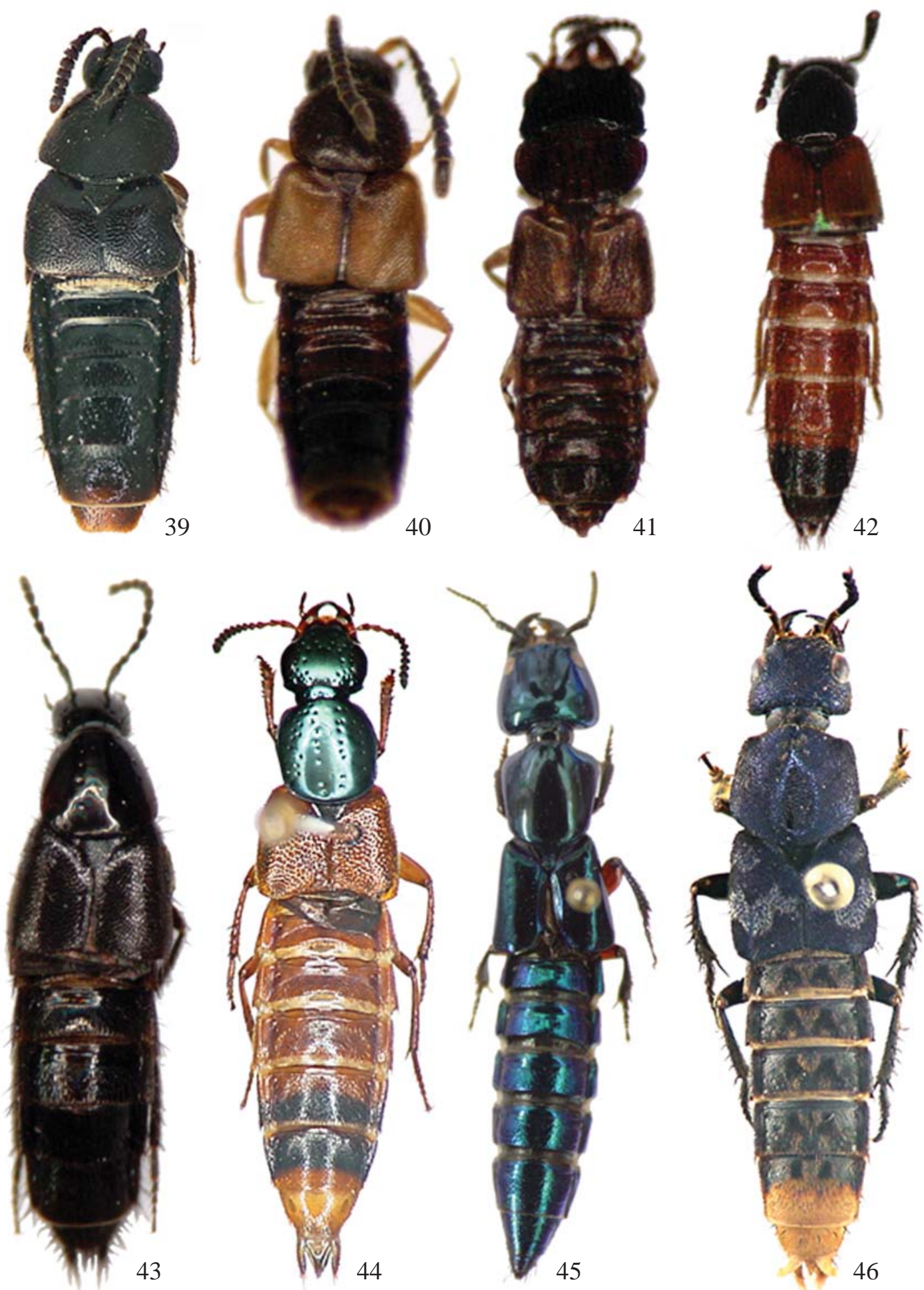

Figs. 39-46. Habitus of Staphylinidae. 39, Aleochara sp.; 40, Atheta sp.; 41, Anotylus sp.; 42, Belonuchus sp.; 43, Philonthus sp.; 44, Xanthopygus bicolor; 45, Eulissus chalybaeus; 46, Platydracus ochropygus.

Pronotum with uniform color or with the maculae rounded, occupying the center of the pronotal disk (Fig. 38) ....... Oxelytrum cayennense (Sturm, 1826)

Key to species of Staphylinidae

1. Antennae inserted before anterior margin of eyes
Antennae inserted between eyes (Fig. 15) .... Aleocharinae Maxilar palpi with five segments, the apical minute (pseudosegment) (Figs. 15 e 39) Aleochara Maxilar palpi with four segments, without pseudosegment (Fig. 40) Atheta 


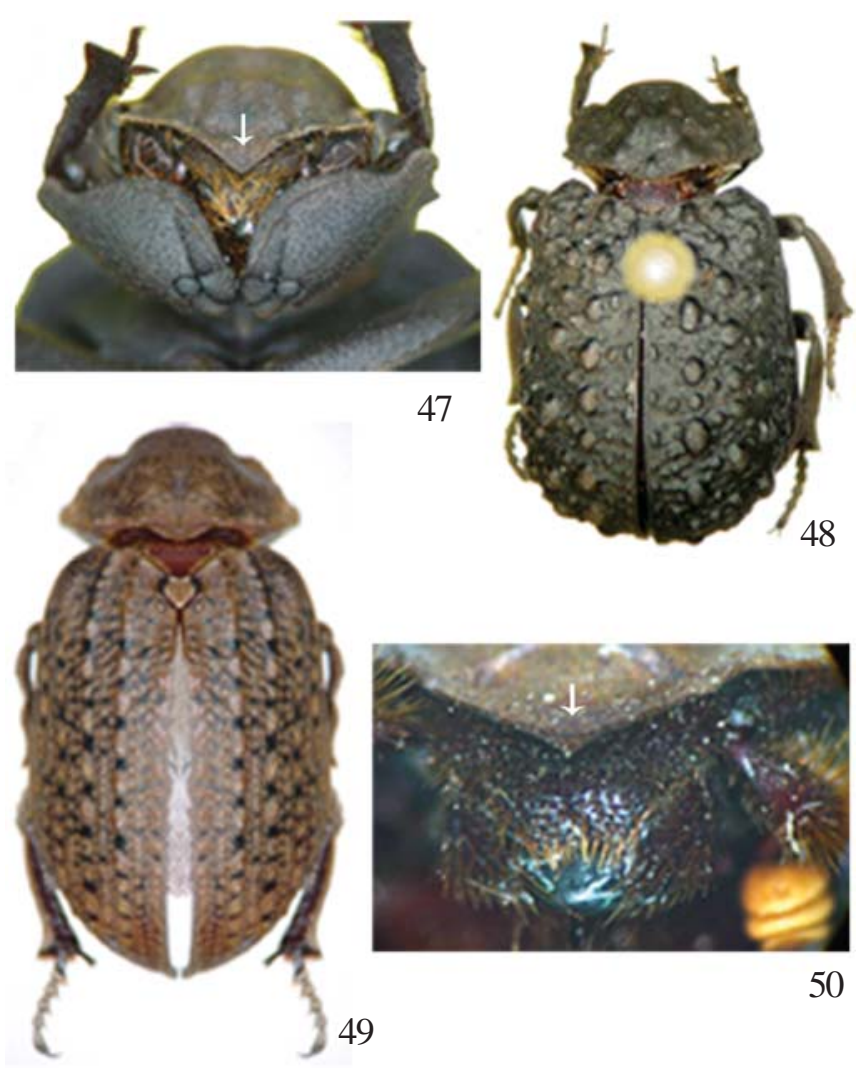

Figs. 47-50. Trogidae. Head, frontal view: 47, Polynonchus sp. (clypeus); Habitus: 48, Polynonchus sp.; 49, Omorgus sp.; head, frontal view: 50, Omorgus sp. (clypeus).

2. Abdomen with seven visible sternites Oxytelinae Scutellum with a diamond shaped impression; abdominal tergum II with curved basal lateral ridge .......... Oxytelus

Scutellum with a tri- or bilobed impression; abdominal tergum II without curved basal lateral ridge (Fig. 41)

Anotylus

2'. Abdomen with six visible sternites ...... Staphylininae

a. The first visible segment of abdominal terga with impressed curved line Xanthopygus

Head and pronotum green bluish colored; elytrae and basal segments of abdomen yellow brownish; size approximately $14 \mathrm{~mm}$ (Fig. 44)

Xanthopygus bicolor (LaPorte, 1835)

a'. Without above combination of characters

b. With sclerotized plate in front of prosternum (Figs. 16) Eulissus

Body green bluish, size approximately $21 \mathrm{~mm}$ (Fig. 45) .... Eulissus chalybaeus (Mannerheim, 1830)

b'. Without sclerotized plate in front of prosternum ....

c. Pronotum with translucent post-coxal process . Platydracus

Head, pronotum and elytrae metallic bluish; abdomen black, with the three last segments yellow colored; size approximately $16 \mathrm{~mm}$ (Fig. 46) .....

Platydracus ochropygus (Nordman, 1837) c'. Without above combination of characters d

d. Apical segment of maxilar palpi twice the size of the penultimate segment (Figs. 17, 42) ............... Belonuchus

d'. Apical segment of maxilar palpi more or less of the same size of the penultimate segment (Figs. 18, 43)

Philonthus

Key to species of Trogidae

1. Clypeus forming a $90^{\circ}$ angle with frons (Figs. 47, 48) Polynoncus

Clypeus not forming a $90^{\circ}$ angle with frons (Figs. 49, 50) Omorgus

Characteristics of families and species

Carabidae. Ground beetles. A family with about 1,500 genera and 30,000 species, with 336 genera and 6,258 species in the Neotropical region. Usually found under stones, logs, leaves, bark and debris. The larvae and adults hide during the day and feed at night, preferring to walk rather than fly. Most of them are predators of other insects and may be used in biological control. Some species feed on dead or dying insects and others on living insects, such as maggots, beetle larvae, etc.

Cleridae. Checkered beetles. A family with about 150 genera and 4,000 species, with 61 genera and 886 species in the Neotropical region. They attack insects specially wood-boring beetles. The adults are very active specially during the day. They are often found on flowers, foliage and tree trunks. Necrobia ruficollis (Fabricius, 1775) and Necrobia rufipes (DeGeer, 1775) occur in carrion and products of animal origin.

Necrobia rufipes is predator of dipteran and coleopteran larvae. It is also associated with stored meats, such as dry fish, skin, dead animal bones, some oily seeds and stored products, mainly those with high protein indices, being also found in animal rations (Gredilha et al. 2005; Ashman 1963).

Dermestidae. Odd beetles. A family with about 45 genera and 850 species, with 20 genera and 248 species in the Neotropical region. Also known as skin beetles, they are primarily scavengers that feed on dried skin and other soft remains of animals such as fur, feathers, wool and leather. They also feed on carpets, silk, dried meats and dead insects. Some are pests of storage products such as grains, seeds, corks or cereal products.

Dermestes maculatus De Geer is a notorious pest of dried fish and fish meal, is known to damage wooden frames as well as polystyrene and glass fibre wadding in premises when the last instar larva is about to pupate (Turner 1986; Wildey \& Wayman 1979). The insect pests of dried animal products also attack living insects Dermestes spp. on silkworm pupae and adults (Kumar et al. 1988; Veer et al. 1996). Use of infested woolen materials can cause allergic reactions like urticarial and papulovesicular lesions in man (Ahmed et al. 1981).

Histeridae. Clown beetles. A family with about 200 genera and 3,000 species, with 139 genera and 1,047 species in the 
Table I. List of the main Coleoptera species of forensic importance from South America and their respective substratum/carcasses, geographic distribution and references.

\begin{tabular}{|c|c|c|c|}
\hline Families/species & $\begin{array}{l}\text { Substratum/ } \\
\text { carcasses }\end{array}$ & Geographic distribution & References \\
\hline \multicolumn{4}{|l|}{ Carabidae } \\
\hline Argutoridius bonariensis (Dejean) & Pig & Buenos Aires, Argentina & Scampini et al. 2002 \\
\hline Bradycellus (Bradycellus) sp. & Pig & Buenos Aires, Argentina & Scampini et al. 2002 \\
\hline Loxandrus confusus (Dejean) & Pig & Buenos Aires, Argentina & Scampini et al. 2002 \\
\hline Loxandrus planicollis Straneo & Pig & Buenos Aires, Argentina & Scampini et al. 2002 \\
\hline Loxandrus simplex (Dejean) & Pig & Buenos Aires, Argentina & Scampini et al. 2002 \\
\hline Taeniolobus crenulatus Chaudoir (?) & general & São Paulo, Brazil & Luederwaldt 1911 \\
\hline $\begin{array}{l}\text { Trirammatus (Feroniomorpha) } \\
\text { striatulus (Fabricius) }\end{array}$ & Pig & Buenos Aires, Argentina & Scampini et al. 2002 \\
\hline Carabidae sp. & $\begin{array}{l}\text { Human } \\
\text { corpses; pig }\end{array}$ & Campinas, Brazil & Carvalho et al. 2000 \\
\hline Carabidae sp. & Pig & Medellín, Colombia & Wolff et al. 2001 \\
\hline Carabidae sp. & Pig & Recife, Brazil & Cruz \& Vasconcelos 2006 \\
\hline Carabidae sp. 1 & Pig & Curitiba, Brazil & Mise et al. 2007 \\
\hline Carabidae sp. 2 & Pig & Curitiba, Brazil & Mise et al. 2007 \\
\hline Carabidae sp.3 & Pig & Curitiba, Brazil & Mise et al. 2007 \\
\hline Carabidae sp.4 & Pig & Curitiba, Brazil & Mise et al. 2007 \\
\hline Carabidae sp. 5 & Pig & Curitiba, Brazil & Mise et al. 2007 \\
\hline \multicolumn{4}{|l|}{ Cleridae } \\
\hline Necrobia ruficollis (Fabricius) & $\begin{array}{l}\text { Pig and other } \\
\text { carcasses }\end{array}$ & $\begin{array}{l}\text { Campinas, São Paulo and Curitiba, } \\
\text { Brazil; Buenos Aires, Argentina }\end{array}$ & $\begin{array}{l}\text { Luederwaldt 1911; Centeno } \text { et al. 2002; } \\
\text { Mise } \text { et al. } 2007\end{array}$ \\
\hline Necrobia rufipes (De Geer) & $\begin{array}{l}\text { Human } \\
\text { corpses; pig } \\
\text { and other } \\
\text { carcasses }\end{array}$ & $\begin{array}{l}\text { São Paulo, Campinas and Curitiba, } \\
\text { Brazil; Medellín, Colombia; Buenos } \\
\text { Aires, Argentina; Callao, Peru }\end{array}$ & $\begin{array}{l}\text { Luederwaldt 1911; Souza \& Linhares } \\
\text { 1997; Carvalho et al. 2000; Wolff et al. } \\
\text { 2001; Centeno et al. 2002; Iannacone } \\
\text { 2003; Carvalho et al. 2004; Mise et } \\
\text { al.2007 }\end{array}$ \\
\hline $\begin{array}{l}\text { Cleridae sp. } \\
\text { Dermestidae }\end{array}$ & Pig & Recife, Brazil & Cruz \& Vasconcelos 2006 \\
\hline Dermestes ater De Geer & Pig & Buenos Aires, Argentina & Centeno et al. 2002 \\
\hline Dermestes carnivorus (Fabricius) & Dog & San José, Costa Rica & Jirón \& Cartín 1981 \\
\hline Dermestes maculatus (De Geer) & $\begin{array}{l}\text { Human } \\
\text { corpses; pig, } \\
\text { rat and other } \\
\text { carcasses }\end{array}$ & $\begin{array}{l}\text { Campinas and Curitiba, Brazil; } \\
\text { Buenos Aires, Argentina; Altos de } \\
\text { Pipe (Caracas), Venezuela; Callao, } \\
\text { Peru }\end{array}$ & $\begin{array}{l}\text { Souza \& Linhares 1997; Carvalho et al. } \\
\text { 2000; Centeno et al. 2002; Iannacone } \\
\text { 2003; Velásquez 2007; Carvalho et al. } \\
\text { 2004; Mise et al. } 2007\end{array}$ \\
\hline Dermestes peruvianus Laporte & Pig & Campinas, Brazil & Souza \& Linhares 1997 \\
\hline Dermestes sp. & Pig & Medellín, Colombia & Wolff et al. 2001 \\
\hline Dermestes sp. & general & São Paulo, Brazil & Luederwaldt 1911 \\
\hline $\begin{array}{l}\text { Geotrupidae } \\
\text { Histeridae }\end{array}$ & Dog & San José, Costa Rica & Jirón \& Cartín 1981 \\
\hline Euspilotus aenicollis Marshall & $\operatorname{Dog}$ & San José, Costa Rica & Jirón \& Cartín 1981 \\
\hline $\begin{array}{l}\text { Euspilotus nigrita (Blanchard) } \\
\text { (=Euspilotus azureus) }\end{array}$ & Pig & Curitiba, Brazil & Mise et al. 2007 \\
\hline Euspilotus sp. & Pig & Campinas, Brazil & Souza \& Linhares 1997 \\
\hline Euspilotus sp. & Pig & Campinas, Brazil & Carvalho et al. 2000 \\
\hline Euspilotus sp. & Pig & Curitiba, Brazil & Mise et al. 2007 \\
\hline $\begin{array}{l}\text { Geomysaprinus (Priscosaprinus) } \\
\text { belioculus (Marseul) }\end{array}$ & Dog & San José, Costa Rica & Jirón \& Cartín 1981 \\
\hline Hister punctiger LeConte & Dog & San José, Costa Rica & Jirón \& Cartín 1981 \\
\hline Hister sp. & Pig & Medellín, Colombia & Wolff et al. 2001 \\
\hline Hister sp. & Pig & Buenos Aires, Argentina & Centeno et al. 2002 \\
\hline Hister sp. & Pig & Curitiba, Brazil & Mise et al. 2007 \\
\hline Saprinus aeneus Fabricius & Pig & Callao, Peru & Iannacone 2003 \\
\hline Saprinus azureus (Sahlberg) & Pig & Campinas, Brazil & Souza \& Linhares 1997 \\
\hline Saprinus patagonicus Blanchard & Pig & Buenos Aires, Argentina & Centeno et al. 2002 \\
\hline Omalodes bifoveolatus (Marseul) & Pig & Manaus, Brazil & in this paper \\
\hline Omalodes sp. & Pig & Campinas, Brazil & Souza \& Linhares 1997 \\
\hline Omalodes sp. & Pig & Campinas, Brazil & Carvalho et al. 2000 \\
\hline Phelister sp. & Pig & Curitiba, Brazil & Mise et al. 2007 \\
\hline
\end{tabular}


Table I. Cont.

\begin{tabular}{|c|c|c|c|}
\hline Families/species & $\begin{array}{l}\text { Substratum/ } \\
\text { carcasses }\end{array}$ & Geographic distribution & References \\
\hline Histeridae sp. & Rat & Campinas, Brazil & Monteiro-Filho \& Penereiro 1987 \\
\hline Histeridae sp. & Pig & Recife, Brazil & Cruz \& Vasconcelos 2006 \\
\hline Histeridae sp. & Rat & Altos de Pipe (Caracas), Venezuela & Velásquez 2007 \\
\hline Histeridae spp. & $\begin{array}{l}\text { Pig and other } \\
\text { carcasses }\end{array}$ & Campinas and São Paulo, Brazil & Luederwaldt 1911; Carvalho et al. 2004 \\
\hline \multicolumn{4}{|l|}{ Hydrophilidae } \\
\hline Hydrophilidae sp.1 & Pig & Curitiba, Brazil & Mise et al. 2007 \\
\hline Hydrophilidae sp.2 & Pig & Curitiba, Brazil & Mise et al. 2007 \\
\hline Hydrophilidae sp.3 & Pig & Curitiba, Brazil & Mise et al. 2007 \\
\hline Hydrophilidae sp.4 & Pig & Curitiba, Brazil & Mise et al. 2007 \\
\hline \multicolumn{4}{|l|}{ Leiodidae (=Cholevidae) } \\
\hline Dissochaetus murray Reitter & Rat & Curitiba, Brazil & Moura et al. 1997 \\
\hline Hydnobius sp. & Pig & Curitiba, Brazil & Mise et al. 2007 \\
\hline $\begin{array}{l}\text { Leiodidae sp. } \\
\text { Nitidulidae }\end{array}$ & Rat & Altos de Pipe (Caracas), Venezuela & Velásquez 2007 \\
\hline Carpophilus sp. & Pig & Curitiba, Brazil & Mise et al. 2007 \\
\hline Nitidulidae sp. & Pig & Medellín, Colombia & Wolff et al. 2001 \\
\hline Nitidulidae sp. & Rat & Campinas, Brazil & Monteiro-Filho \& Penereiro 1987 \\
\hline Nitidulidae sp.1 & Pig & Curitiba, Brazil & Mise et al. 2007 \\
\hline Nitidulidae sp.2 & Pig & Curitiba, Brazil & Mise et al. 2007 \\
\hline Nitidulidae sp.3 & Pig & Curitiba, Brazil & Mise et al. 2007 \\
\hline \multicolumn{4}{|l|}{ Ptiliidae } \\
\hline Ptiliidae sp. & Pig & Curitiba, Brazil & Mise et al. 2007 \\
\hline Rhizophagidae & & & \\
\hline Rhizophagidae sp. & Pig & Curitiba, Brazil & Mise et al. 2007 \\
\hline \multicolumn{4}{|l|}{ Scarabaeidae } \\
\hline Aphengium sordidum Harold & general & São Paulo and neighborhood, Brazil & Pessôa \& Lane 1941 \\
\hline Ataenius picinus Harold & Pig & Curitiba, Brazil & Mise et al. 2007 \\
\hline Bdelyrus lagopus (Harold) & general & São Paulo and neighborhood, Brazil & Pessôa \& Lane 1941 \\
\hline Boucomontius convexus Harold & general & São Paulo and neighborhood, Brazil & Pessôa \& Lane 1941 \\
\hline $\begin{array}{l}\text { Canthidium apicatum (Harold) } \\
\text { (=Ateuchus apicatum) }\end{array}$ & general & São Paulo, Brazil & Luederwaldt 1911 \\
\hline Canthidium breve (Germar) & general & São Paulo and neighborhood, Brazil & Luederwaldt 1911; Pessôa \& Lane 1941 \\
\hline Canthidium decoratum Perty & general & São Paulo and neighborhood, Brazil & Luederwaldt 1911; Pessôa \& Lane 1941 \\
\hline Canthidium dispar Harold & general & São Paulo and neighborhood, Brazil & Luederwaldt 1911; Pessôa \& Lane 1941 \\
\hline Canthidium lucidum Harold & general & São Paulo and neighborhood, Brazil & Pessôa \& Lane 1941 \\
\hline Canthidium politum Politum (?) & general & São Paulo, Brazil & Luederwaldt 1911 \\
\hline $\begin{array}{l}\text { Canthidium splendidum Preudhomme de } \\
\text { Borre }\end{array}$ & general & São Paulo and neighborhood, Brazil & Luederwaldt 1911; Pessôa \& Lane 1941 \\
\hline Canthidium sp. & Rat & Curitiba, Brazil & Moura et al. 1997 \\
\hline Canthidium sp. & general & São Paulo and neighborhood, Brazil & Pessôa \& Lane 1941 \\
\hline Canthidium spp. & general & São Paulo, Brazil & Luederwaldt 1911 \\
\hline Canthon angularis Harold & general & São Paulo and neighborhood, Brazil & Pessôa \& Lane 1941 \\
\hline Canthon conformisHarold & general & São Paulo and neighborhood, Brazil & Luederwaldt 1911; Pessôa \& Lane 1941 \\
\hline Canthon curvipes Harold & general & São Paulo and neighborhood, Brazil & Luederwaldt 1911; Pessôa \& Lane 1941 \\
\hline Canthon dives Harold & general & São Paulo and neighborhood, Brazil & Luederwaldt 1911; Pessôa \& Lane 1941 \\
\hline Canthon lituratus (Germar) & general & São Paulo and neighborhood, Brazil & Pessôa \& Lane 1941 \\
\hline Canthon mutabilis Lucas & general & São Paulo and neighborhood, Brazil & Pessôa \& Lane 1941 \\
\hline Canthon muticus Harold & general & São Paulo and neighborhood, Brazil & Pessôa \& Lane 1941 \\
\hline Canthon nigriceps Harold & general & São Paulo and neighborhood, Brazil & Pessôa \& Lane 1941 \\
\hline Canthon opacus Lucas & general & São Paulo and neighborhood, Brazil & Pessôa \& Lane 1941 \\
\hline Canthon podagricus (Harold) & general & São Paulo and neighborhood, Brazil & Luederwaldt 1911; Pessôa \& Lane 1941 \\
\hline Canthon rutilans Laporte & $\begin{array}{l}\text { Rat and other } \\
\text { carcasses }\end{array}$ & $\begin{array}{l}\text { São Paulo and neighborhood, } \\
\text { Campinas, Brazil }\end{array}$ & $\begin{array}{l}\text { Luederwaldt 1911; Pessôa \& Lane 1941; } \\
\text { Monteiro-Filho \& Penereiro } 1987\end{array}$ \\
\hline Canthon septemmaculatum (Latreille) & general & São Paulo and neighborhood, Brazil & Luederwaldt 1911; Pessôa \& Lane 1941 \\
\hline Canthon smaragdulus (Fabricius) & general & São Paulo and neighborhood, Brazil & Pessôa \& Lane 1941 \\
\hline Canthon speculifer Laporte & general & São Paulo and neighborhood, Brazil & Pessôa \& Lane 1941 \\
\hline Canthon substriatus Harold & general & São Paulo and neighborhood, Brazil & Pessôa \& Lane 1941 \\
\hline Canthon tetraodon Blanchard & general & São Paulo and neighborhood, Brazil & Pessôa \& Lane 1941 \\
\hline
\end{tabular}


Table I. Cont.

\begin{tabular}{|c|c|c|c|}
\hline Families/species & $\begin{array}{l}\text { Substratum/ } \\
\text { carcasses }\end{array}$ & Geographic distribution & References \\
\hline Canthon triangularis (Drury) & general & São Paulo and neighborhood, Brazil & Pessôa \& Lane 1941 \\
\hline Canthon tristis Harold & general & São Paulo, Brazil & Luederwaldt 1911 \\
\hline Canthon virens Mannerheim & general & São Paulo and neighborhood, Brazil & Pessôa \& Lane 1941 \\
\hline Canthon sp. & Pig & Campinas, Brazil & Carvalho et al. 2000 \\
\hline Chalcocopris hesperus (Olivier) & general & São Paulo and neighborhood, Brazil & Pessôa \& Lane 1941 \\
\hline Choeridium breve Harold & general & São Paulo and neighborhood, Brazil & Pessôa \& Lane 1941 \\
\hline Choeridium carbonarium Harold & general & São Paulo and neighborhood, Brazil & Pessôa \& Lane 1941 \\
\hline Choeridium mutilatum Harold & general & São Paulo and neighborhood, Brazil & Pessôa \& Lane 1941 \\
\hline Choeridium pauperatum (Germar) & general & São Paulo and neighborhood, Brazil & Luederwaldt 1911; Pessôa \& Lane 1941 \\
\hline $\begin{array}{l}\text { Choeridium striatulum Preudhomme de } \\
\text { Borre }\end{array}$ & general & São Paulo and neighborhood, Brazil & Pessôa \& Lane 1941 \\
\hline Choeridium subquadratum Harold & general & São Paulo and neighborhood, Brazil & Pessôa \& Lane 1941 \\
\hline Choeridium vividum (Germar) & general & São Paulo and neighborhood, Brazil & Pessôa \& Lane 1941 \\
\hline Coprophanaeus arrowi Olsoufieff & general & São Paulo and neighborhood, Brazil & Pessôa \& Lane 1941 \\
\hline Coprophanaeus camargoi Pessôa & general & São Paulo and neighborhood, Brazil & Pessôa \& Lane 1941 \\
\hline Coprophanaeus cerberus Harold & general & São Paulo and neighborhood, Brazil & Pessôa \& Lane 1941 \\
\hline Coprophanaeus cyanescens Olsoufieff & general & São Paulo and neighborhood, Brazil & Pessôa \& Lane 1941 \\
\hline Coprophanaeus dardanus MacLeay & general & São Paulo and neighborhood, Brazil & Pessôa \& Lane 1941 \\
\hline Coprophanaeus ensifer (Germar) & $\begin{array}{l}\text { Pig and other } \\
\text { carcasses }\end{array}$ & $\begin{array}{l}\text { São Paulo and neighborhood, } \\
\text { Campinas, Brazil }\end{array}$ & $\begin{array}{l}\text { Pessôa \& Lane 1941; Carvalho et al. } \\
2000\end{array}$ \\
\hline Coprophanaeus jasius Olivier & general & São Paulo and neighborhood, Brazil & Pessôa \& Lane 1941 \\
\hline Coprophanaeus milon Blanchard & general & São Paulo and neighborhood, Brazil & Pessôa \& Lane 1941 \\
\hline Coprophanaeus punctatus Olsoufieff & general & São Paulo and neighborhood, Brazil & Pessôa \& Lane 1941 \\
\hline Coprophanaeus spitzi Pessôa & general & São Paulo and neighborhood, Brazil & Pessôa \& Lane 1941 \\
\hline Coprophanaeus thalassinus Perti & general & São Paulo and neighborhood, Brazil & Pessôa \& Lane 1941 \\
\hline Coprophanaeus sp. & Pig & Medellín, Colombia & Wolff et al. 2001 \\
\hline Deltochilum brasiliensis Laporte & $\begin{array}{l}\text { Human } \\
\text { corpses; pig }\end{array}$ & $\begin{array}{l}\text { São Paulo and neighborhood, } \\
\text { Campinas, Brazil }\end{array}$ & $\begin{array}{l}\text { Luederwaldt 1911; Pessôa \& Lane 1941; } \\
\text { Carvalho } \text { et al. } 2000\end{array}$ \\
\hline
\end{tabular}

Deltochilum carinatum Westwood Deltochilum dentipes Eschscholtz Deltochilum furcatum (Laporte) Deltochilum icarus (Olivier) Deltochilum ivoratum (Laporte) Deltochilum morbillosum Burmeister Deltochilum orbiculare Lansberge Deltochilum rubripenne Gory Deltochilum sulphuratum Deltochilum trisignatum Harold Deltochilum sp.

Deltorrhinum sp.

Dendropaemon crenatostriatus Felsche Dendropaemon denticollis Felsche Dendropaemon fractipes Felsche Dendropaemon ganglbaueri Felsche Dendropaemon hirticollis Olsoufieff Dendropaemon montei Olsoufieff Dendropaemon viridipennis Laporte Dendropaemon viridis Perty Dendropaemon waterhousei Olsoufieff Dichotomius sericeus Harold

Dichotomius sp.

Dichotomius sp.

Dyscinetus sp.

Eudinopus dytiscoides (Schreibers) Eurysternus calligrammus Dalman Eurysternus femoralis Lucas Eurysternus foedus Guérin and other

carcasses

general

general

general

general

general

general

general

general

general

general

Rat

general

general

general

general

general

general

general

general

general

general

Pig

Pig

Pig

Pig

general

general

general general
São Paulo and neighborhood, Brazil Pessôa \& Lane 1941

São Paulo and neighborhood, Brazil Pessôa \& Lane 1941

São Paulo and neighborhood, Brazil Pessôa \& Lane 1941

São Paulo and neighborhood, Brazil Pessôa \& Lane 1941

São Paulo and neighborhood, Brazil Pessôa \& Lane 1941

São Paulo and neighborhood, Brazil Luederwaldt 1911; Pessôa \& Lane 1941

São Paulo and neighborhood, Brazil Pessôa \& Lane 1941

São Paulo and neighborhood, Brazil Pessôa \& Lane 1941

São Paulo, Brazil

Luederwaldt 1911

São Paulo and neighborhood, Brazil Pessôa \& Lane 1941

Altos de Pipe (Caracas), Venezuela; Velásquez, 2007

São Paulo and neighborhood, Brazil Pessôa \& Lane 1941

São Paulo and neighborhood, Brazil Pessôa \& Lane 1941

São Paulo and neighborhood, Brazil Pessôa \& Lane 1941

São Paulo and neighborhood, Brazil Pessôa \& Lane 1941

São Paulo and neighborhood, Brazil Pessôa \& Lane 1941

São Paulo and neighborhood, Brazil Pessôa \& Lane 1941

São Paulo and neighborhood, Brazil Pessôa \& Lane 1941

São Paulo and neighborhood, Brazil Pessôa \& Lane 1941

São Paulo and neighborhood, Brazil Pessôa \& Lane 1941

São Paulo and neighborhood, Brazil Pessôa \& Lane 1941

Recife, Brazil

Recife, Brazil

Curitiba, Brazil

Curitiba, Brazil

Cruz \& Vasconcelos 2006

Cruz \& Vasconcelos 2006

Mise et al. 2007

Mise et al. 2007

São Paulo and neighborhood, Brazil Pessôa \& Lane 1941

São Paulo and neighborhood, Brazil Pessôa \& Lane 1941

São Paulo and neighborhood, Brazil Pessôa \& Lane 1941

São Paulo and neighborhood, Brazil Pessôa \& Lane 1941 
Table I. Cont

\begin{tabular}{llll}
\hline Families/species & $\begin{array}{l}\text { Substratum/ } \\
\text { carcasses }\end{array}$ & Geographic distribution & References \\
\hline
\end{tabular}

Eurysternus impressicollis Laporte general

Eurysternus opacus Lucas

Eurysternus parallelus Laporte

Eurysternus scotinoides Laporte

Euristernus nr.velutinus (sic)

Eurysternus sp.

Gromphas lacordairei Brullé

Holocephalus eridanus (Olivier)

Leucotyreus sp. (=Leucothyreus sp.)

Megathopa aenicollis Waterhouse

Megathopa virens Harold

Megathopa sp.

Megathopa sp.

Ontherus appendiculatus (Mannerheim)

Ontherus cephalotes Harold

Ontherus digitatus Harold

Ontherus nisus (Laporte)

Ontherus sp.

Ontherus sp.

Onthocharis sp.

Onthophagus bidentatus Drapiez

Onthophagus buculus Mannerheim

Onthophagus hirculus Mannerheim

Onthophagus sp.

Oxysternon conspicillatum Weber

Oxysternon curvispinum Olsoufieff

Oxysternon palaemon Laporte

Pedaridium hirsutum (Harold)

Phanaeus (Phanaeus) dejeani Harold

Phanaeus (Phanaeus) faunus Fabricius

Phanaeus (Phanaeus) floriger Kirby

Phanaeus (Phanaeus) kirbyi Vigors

Phanaeus (Phanaeus) palaeno Blanchard general

Phanaeus (Phanaeus) splendidus general

(Fabricius)

Phanaeus bellicosus (Olivier)

(=Coprophanaeus bellicosus)

Phanaeus bonariensis Gory

(=Coprophanaeus bonariensis)

Phanaeus horus Waterhouse

(=Coprophanaeus horus)

Phanaeus saphirinus Sturm

(=Coprophanaeus saphirinus)

Pinotus agesilaus Waterhouse

Pinotus ascanius Harold

Pinotus fissus Harold

Pinotus inhiatus Germar

Pinotus longiceps Taschenberg

Pinotus nisus (Olivier)

Pinotus semiaeneus Germar

Pinotus smaragdinus (Perty)

Pinotus sp.

Scatimus bicarenatus Harold

Scatonomus fasciculatus Erichson

Scatonomus insignis Harold

Scybalocanthon sp. general

Human

corpses; pig

and other

carcasses

general

Rat

Rat

general

general

Pig

general

general

Rat

Rat

general

general

general

general

Pig

general

general

general

Pig

general

Pig

general

general

general

general

general

general

general

general

general

general

general

Rat and other carcasses

general

general

general

general

general

general

general

general

Rat

general

general

general

Pig
São Paulo and neighborhood, Brazil Luederwaldt 1911; Pessôa \& Lane 1941

São Paulo and neighborhood, Brazil Pessôa \& Lane 1941

São Paulo and neighborhood,

Campinas, Brazil

Pessôa \& Lane 1941; Carvalho et al. 2000

São Paulo and neighborhood, Brazil Pessôa \& Lane 1941

Altos de Pipe (Caracas), Venezuela; Velásquez, 2007

Curitiba, Brazil

Moura et al. 1997

São Paulo and neighborhood, Brazil Pessôa \& Lane 1941

São Paulo and neighborhood, Brazil Pessôa \& Lane 1941

Curitiba, Brazil

Mise et al. 2007

São Paulo and neighborhood, Brazil Pessôa \& Lane 1941

São Paulo and neighborhood, Brazil Pessôa \& Lane 1941

Campinas, Brazil

Curitiba, Brazil

Monteiro-Filho \& Penereiro 1987

Moura et al. 1997

São Paulo and neighborhood, Brazil Pessôa \& Lane 1941

São Paulo and neighborhood, Brazil Pessôa \& Lane 1941

São Paulo and neighborhood, Brazil Pessôa \& Lane 1941

São Paulo and neighborhood, Brazil Pessôa \& Lane 1941

São Paulo, Brazil

Curitiba, Brazil

Luederwaldt 1911

Mise et al. 2007

São Paulo and neighborhood, Brazil Pessôa \& Lane 1941

São Paulo, Brazil

Campinas, Brazil

São Paulo, Brazil

Curitiba, Brazil

Luederwaldt 1911

Souza \& Linhares 1997

Luederwaldt 1911

Mise et al. 2007

São Paulo and neighborhood, Brazil Pessôa \& Lane 1941

São Paulo and neighborhood, Brazil Pessôa \& Lane 1941

São Paulo and neighborhood, Brazil Pessôa \& Lane 1941

São Paulo and neighborhood, Brazil Pessôa \& Lane 1941

São Paulo and neighborhood, Brazil Pessôa \& Lane 1941

São Paulo and neighborhood, Brazil Pessôa \& Lane 1941

São Paulo and neighborhood, Brazil Pessôa \& Lane 1941

São Paulo and neighborhood, Brazil Pessôa \& Lane 1941

São Paulo and neighborhood, Brazil Pessôa \& Lane 1941

São Paulo and neighborhood, Brazil Pessôa \& Lane 1941

São Paulo and neighborhood, Brazil Pessôa \& Lane 1941

São Paulo and neighborhood, Brazil Luederwaldt 1911; Pessôa \& Lane 1941

São Paulo and neighborhood, Brazil Pessôa \& Lane 1941

São Paulo and neighborhood,

Curitiba, Brazil

São Paulo and neighborhood, Brazil

São Paulo, Brazil

Pessôa \& Lane 1941

São Paulo and neighborhood, Brazil Pessôa \& Lane 1941

São Paulo and neighborhood, Brazil Pessôa \& Lane 1941

São Paulo and neighborhood, Brazil Pessôa \& Lane 1941

São Paulo and neighborhood, Brazil Pessôa \& Lane 1941

São Paulo, Brazil

Luederwaldt 1911

São Paulo and neighborhood, Brazil Pessôa \& Lane 1941

Curitiba, Brazil

Moura et al. 1997

São Paulo and neighborhood, Brazil Pessôa \& Lane 1941

São Paulo and neighborhood, Brazil Pessôa \& Lane 1941

São Paulo and neighborhood, Brazil Pessôa \& Lane 1941

Campinas, Brazil

Carvalho et al. 2000 
Table I. Cont.

\begin{tabular}{|c|c|c|c|}
\hline Families/species & $\begin{array}{l}\text { Substratum/ } \\
\text { carcasses }\end{array}$ & Geographic distribution & References \\
\hline Taurocopris luderwaldti Pessôa & general & São Paulo and neighborhood, Brazil & Pessôa \& Lane 1941 \\
\hline Taurocopris mimas Linnaeus & general & São Paulo and neighborhood, Brazil & Pessôa \& Lane 1941 \\
\hline Taurocopris mirabilis Harold & general & São Paulo and neighborhood, Brazil & Pessôa \& Lane 1941 \\
\hline Trichillum externepunctatum & general & São Paulo and neighborhood, Brazil & Pessôa \& Lane 1941 \\
\hline \multicolumn{4}{|l|}{ Preudhomme de Borre } \\
\hline Trichillum heydeni Harold & general & São Paulo and neighborhood, Brazil & Luederwaldt 1911; Pessôa \& Lane 1941 \\
\hline Trichillum hirsutum Boucomont & general & São Paulo and neighborhood, Brazil & Pessôa \& Lane 1941 \\
\hline Trichillum sp. & general & São Paulo, Brazil & Luederwaldt 1911 \\
\hline Uroxys metallecens Harold & general & São Paulo and neighborhood, Brazil & Pessôa \& Lane 1941 \\
\hline Aphodiinae sp.1 & Pig & Curitiba, Brazil & Mise et al. 2007 \\
\hline Scarabaeidae sp. & Pig & Medellín, Colombia & Wolff et al. 2001 \\
\hline Scarabaeidae spp. & Pig & Campinas, Brazil & Carvalho et al. 2004 \\
\hline
\end{tabular}

Silphidae

Hyponecrodes sp.

Oxelytrum discicolle (Brulle)

Oxyletrum discicolle (sic)

Oxyletrum disciolle (sic)

Oxelytrum sp.

Silpha cayennensis (Sturm)

(=Oxelytrum cayennense)

Silphidae sp.

Silphidae sp.

Staphylinidae

Achenomorphus sp.

Aleochara lateralis Erichson

Aleochara notula Erichson

Aleochara taeniata Erichson

Aleochara sp.1 (=Aleochara

pseudochrysorrhoa)

Aleochara sp. 2

Aleochara sp. 3

Amblyopinus gahani Fauvel

Anotylus aff. fragilis

Anotylus aff. insignitus

Anotylus aff. nanus

Anotylus aff. spinifrons

Anotylus sp.1

Anotylus sp.1

Anotylus sp.2

Anotylus sp.2

Astenus sp.

Atheta brasiliana Bernhauer

Atheta luederwaldti (Bernhauer)

(=Stethusa luederwaldti)

Atheta lurida (Erichson) (=Stethusa

lurida)

Atheta mayalis Bernhauer

Baeocera sp.1

Baeocera sp.2

Belonuchus apiciventris (Sharp)

Belonuchus basiventris (Sharp)

Belonuchus oxyporinus (Sharp)

Belonuchus pollens Sharp
Pig
Human
cadaver

cadaver; rat

and pig

Rat

Pig

Pig

general

Rat

Dog

Squid

Pig

general

general

Pig

Pig

Pig

general

Squid

Squid

Squid

Squid

Squid

Pig

Squid

Pig

Squid

general

general

general

general

Squid

Squid

Squid

Squid

\section{Buenos Aires, Argentina}

Cali, Colombia; Altos de Pipe

(Caracas), Venezuela;Curitiba,

Brazil

Curitiba, Brazil

Campinas, Brazil

Medellín, Colombia

São Paulo, Brazil

Campinas, Brazil

San José, Costa Rica

Tejupilco, Mexico

Campinas; São Paulo, Brazil

São Paulo, Brazil

São Paulo, Brazil

Curitiba, Brazil

Curitiba, Brazil

Curitiba, Brazil

São Paulo, Brazil

Tlayacapan, Mexico

Tlayacapan, Mexico

Tlayacapan, Mexico

Tejupilco, Mexico

Tejupilco, Mexico

Curitiba, Brazil

Tejupilco, Mexico

Curitiba, Brazil

Tejupilco, Mexico

São Paulo, Brazil

São Paulo, Brazil

São Paulo, Brazil

São Paulo, Brazil

Tlayacapan, Mexico

Tlayacapan, Mexico

Tlayacapan and Tejupilco, Mexico

Tlayacapan and Tejupilco, Mexico

Squid

Squid
Centeno et al. 2002

Barreto et al. 2002; Velásquez 2007;

Mise et al. 2007

Moura et al. 1997

Carvalho et al. 2000

Wolff et al. 2001

Luederwaldt 1911

Monteiro-Filho \& Penereiro 1987

Jirón \& Cartín 1981

Jiménez-Sánchez et al. 2000

Luederwaldt 1911 and Souza \& Linhares 1997

Luederwaldt 1911

Luederwaldt 1911

Mise et al. 2007

Mise et al. 2007

Mise et al. 2007

Luederwaldt 1911

Márquez-Luna 2001

Márquez-Luna 2001

Márquez-Luna 2001

Jiménez-Sánchez et al. 2000

Jiménez-Sánchez et al. 2000

Mise et al. 2007

Jiménez-Sánchez et al. 2000

Mise et al. 2007

Jiménez-Sánchez et al. 2000

Luederwaldt 1911

Luederwaldt 1911

Luederwaldt 1911

Luederwaldt 1911

Márquez-Luna 2001

Márquez-Luna 2001

Jiménez-Sánchez et al. 2000; MárquezLuna 2001

Jiménez-Sánchez et al. 2000; Márquez-

Luna 2001

Tlayacapan and Tejupilco, Mexico Jiménez-Sánchez et al. 2000; MárquezLuna 2001

Tlayacapan and Tejupilco, Mexico Jiménez-Sánchez et al. 2000; Márquez- 
Table I. Cont.

\begin{tabular}{|c|c|c|c|}
\hline Families/species & $\begin{array}{l}\text { Substratum/ } \\
\text { carcasses }\end{array}$ & Geographic distribution & References \\
\hline Belonuchus rufipennis (Fabricius) & Squid & Tlayacapan and Tejupilco, Mexico & $\begin{array}{l}\text { Luna } 2001 \\
\text { Jiménez-Sánchez et al. 2000; Márquez- } \\
\text { Luna } 2001\end{array}$ \\
\hline Belonuchus trochanterinus (Sharp) & Squid & Tlayacapan, Mexico & Márquez-Luna 2001 \\
\hline Belonuchus viridipennis Baudi & Squid & Tlayacapan, Mexico & Márquez-Luna 2001 \\
\hline Belonuchus xanthomelas Solsky & Squid & Tlayacapan and Tejupilco, Mexico & $\begin{array}{l}\text { Jiménez-Sánchez et al. 2000; Márquez- } \\
\text { Luna } 2001\end{array}$ \\
\hline Belonuchus xanthopus Solsky & general & São Paulo, Brazil & Luederwaldt 1911 \\
\hline Belonuchus sp. "group" rufipennis & Squid & Tlayacapan, Mexico & Márquez-Luna 2001 \\
\hline Belonuchus sp.1 & Squid & Tlayacapan, Mexico & Márquez-Luna 2001 \\
\hline Belonuchus sp.2 & Squid & Tlayacapan, Mexico & Márquez-Luna 2001 \\
\hline Belonuchus sp. & Pig & Curitiba, Brazil & Mise et al. 2007 \\
\hline Belonuchus sp. & Squid & Tejupilco, Mexico & Jiménez-Sánchez et al. 2000 \\
\hline Biocrypta sp. & Squid & Tejupilco, Mexico & Jiménez-Sánchez et al. 2000 \\
\hline Bryoporus sp. & Squid & Tejupilco, Mexico & Jiménez-Sánchez et al. 2000 \\
\hline Bryoporus sp. & Squid & Tlayacapan, Mexico & Márquez-Luna 2001 \\
\hline Carpelinus sp. & Pig & Curitiba, Brazil & Mise et al. 2007 \\
\hline Chroaptomus flagrans (Erichson) & Squid & Tlayacapan and Tejupilco, Mexico & $\begin{array}{l}\text { Jiménez-Sánchez et al. 2000; Márquez- } \\
\text { Luna } 2001\end{array}$ \\
\hline Cilea sp. & Pig & Curitiba, Brazil & Mise et al. 2007 \\
\hline Coproporus hepaticus (Erichson) & Squid & Tlayacapan and Tejupilco, Mexico & $\begin{array}{l}\text { Jiménez-Sánchez et al. 2000; Márquez- } \\
\text { Luna } 2001\end{array}$ \\
\hline Coproporus aff. arizonae & Squid & Tlayacapan, Mexico & Márquez-Luna 2001 \\
\hline Coproporus aff. segnis & Squid & Tlayacapan, Mexico & Márquez-Luna 2001 \\
\hline Coproporus sp. & Squid & Tejupilco, Mexico & Jiménez-Sánchez et al. 2000 \\
\hline Creophilus maxillosus (Linnaeus) & Pig and squid & $\begin{array}{l}\text { Tlayacapan, Mexico; Buenos } \\
\text { Aires, Argentina }\end{array}$ & $\begin{array}{l}\text { Márquez-Luna 2001; Centeno } \text { et al. } \\
2002\end{array}$ \\
\hline Creophilus variegatus (Linnaeus) & general & São Paulo, Brazil & Luederwaldt 1911 \\
\hline Cyparium aff. terminale & Squid & Tlayacapan, Mexico & Márquez-Luna 2001 \\
\hline Dibelonetes sp. & Squid & Tlayacapan, Mexico & Márquez-Luna 2001 \\
\hline Echiaster sp. & Pig & Curitiba, Brazil & Mise et al. 2007 \\
\hline Eleusis bicolor Erichson & Squid & Tlayacapan, Mexico & Márquez-Luna 2001 \\
\hline Eleusis sp. & Squid & Tejupilco, Mexico & Jiménez-Sánchez et al. 2000 \\
\hline Eulissus chalibaeus (Mannerheim) & Pig & Campinas, Brazil & Souza \& Linhares 1997 \\
\hline Eulissus sp. & Pig & Curitiba, Brazil & Mise et al. 2007 \\
\hline Falagria fissula (Erichson) & general & São Paulo, Brazil & Luederwaldt 1911 \\
\hline Gabrius sp. & Squid & Tlayacapan, Mexico & Márquez-Luna 2001 \\
\hline $\begin{array}{l}\text { Gastrisus newtonorum Navarrete \& } \\
\text { Márquez }\end{array}$ & Squid & Tlayacapan and Tejupilco, Mexico & $\begin{array}{l}\text { Jiménez-Sánchez et al. 2000; Márquez- } \\
\text { Luna } 2001\end{array}$ \\
\hline Hamotus sp. & Squid & Tlayacapan, Mexico & Márquez-Luna 2001 \\
\hline Heterothops boops Bernhauer & Squid & Tlayacapan, Mexico & Márquez-Luna 2001 \\
\hline Heterothops tenuicornis Sharp & Squid & Tlayacapan, Mexico & Márquez-Luna 2001 \\
\hline Heterotops sp. (=Heterothops sp.) & Pig & Curitiba, Brazil & Mise et al. 2007 \\
\hline Hipotelus sp. & Pig & Medellín, Colombia & Wolff et al. 2001 \\
\hline Homaeotarsus sp. & Squid & Tejupilco, Mexico & Jiménez-Sánchez et al. 2000 \\
\hline Hoplandria aleocharoides Bernhauer & general & São Paulo, Brazil & Luederwaldt 1911 \\
\hline Ischnosoma ashei Campbell & Squid & Tlayacapan, Mexico & Márquez-Luna 2001 \\
\hline Jubomorphus sp. & Squid & Tlayacapan, Mexico & Márquez-Luna 2001 \\
\hline Lepitacnus sp. & Pig & Curitiba, Brazil & Mise et al. 2007 \\
\hline Lispinus sp. & Pig & Medellín, Colombia & Wolff et al. 2001 \\
\hline Lissohypnus sp. & Pig & Curitiba, Brazil & Mise et al. 2007 \\
\hline Lordithon antennatus Campbell & Squid & Tlayacapan, Mexico & Márquez-Luna 2001 \\
\hline Lordithon howdeni Campbell & Squid & Tlayacapan, Mexico & Márquez-Luna 2001 \\
\hline Megalopinus sp. & Pig & Medellín, Colombia & Wolff et al. 2001 \\
\hline Megarthrus aff. altivagans & Squid & Tlayacapan, Mexico & Márquez-Luna 2001 \\
\hline Megarthrus sp. & Squid & Tejupilco, Mexico & Jiménez-Sánchez et al. 2000 \\
\hline Monista sp. & Squid & Tlayacapan, Mexico & Márquez-Luna 2001 \\
\hline Nacaeus sp. & Pig & Curitiba, Brazil & Mise et al. 2007 \\
\hline Neohypnus championi (Sharp) & Squid & Tlayacapan, Mexico & Márquez-Luna 2001 \\
\hline
\end{tabular}


Table I. Cont.

\begin{tabular}{|c|c|c|c|}
\hline Families/species & $\begin{array}{l}\text { Substratum/ } \\
\text { carcasses }\end{array}$ & Geographic distribution & References \\
\hline Neohypnus sp. & Squid & Tlayacapan, Mexico; & Márquez-Luna 2001 \\
\hline Neohypnus sp. & Pig & Curitiba, Brazil & Mise et al. 2007 \\
\hline Ocalea sp.1 (sic) Atheta iheringi & Pig & Curitiba, Brazil & Mise et al. 2007 \\
\hline Ocalea sp.2 & Pig & Curitiba, Brazil & Mise et al. 2007 \\
\hline Osorius sp. & Squid & Tejupilco, Mexico & Jiménez-Sánchez et al. 2000 \\
\hline Oxytelus laqueatus (Marsham) & Squid & Tlayacapan, Mexico & Márquez-Luna 2001 \\
\hline Oxytelus subnitidus Bernhauer & general & São Paulo, Brazil & Luederwaldt 1911 \\
\hline Paederomimus angularius (Erichson) & Squid & Tlayacapan and Tejupilco, Mexico & $\begin{array}{l}\text { Jiménez-Sánchez et al. 2000; Márquez- } \\
\text { Luna } 2001\end{array}$ \\
\hline Paederomimus gentillis Sharp & Squid & Tlayacapan and Tejupilco, Mexico & $\begin{array}{l}\text { Jiménez-Sánchez et al. 2000; Márquez- } \\
\text { Luna } 2001\end{array}$ \\
\hline Paederominus sp. 1 & Squid & Tejupilco, Mexico & Jiménez-Sánchez et al. 2000 \\
\hline Paederus sp. & Squid & Tejupilco, Mexico & Jiménez-Sánchez et al. 2000 \\
\hline Paederus sp. & Pig & Buenos Aires, Argentina & Centeno et al. 2002 \\
\hline Philonthus brasilianus Bernhauer & general & São Paulo, Brazil & Luederwaldt 1911 \\
\hline Philonthus ferialis Erichson (sic) & general & São Paulo, Brazil & Luederwaldt 1911 \\
\hline Philonthus flavolimbatus Erichson & general & São Paulo, Brazil & Luederwaldt 1911 \\
\hline Philonthus iris Sharp & Squid & Tlayacapan, Mexico & Márquez-Luna 2001 \\
\hline Philonthus longicornis Stephens & Pig and squid & Tlayacapan, Mexico & Márquez-Luna 2001 \\
\hline Philonthus piceatus Nordman & Squid & Tlayacapan, Mexico & Márquez-Luna 2001 \\
\hline Philonthus sericans (Gravenhorst) & Squid & Tlayacapan, Mexico & Márquez-Luna 2001 \\
\hline Philonthus testaceipennis Erichson & Squid & Tlayacapan, Mexico & Márquez-Luna 2001 \\
\hline Philonthus aff. apheles & Squid & Tlayacapan, Mexico & Márquez-Luna 2001 \\
\hline Philonthus sp.1 & Pig & Campinas, Brazil & Souza \& Linhares 1997 \\
\hline Philonthus sp.1 & Squid & Tlayacapan, Mexico & Márquez-Luna 2001 \\
\hline Philonthus sp.1 & Pig & Curitiba, Brazil & Mise et al. 2007 \\
\hline Philonthus sp.1 & Squid & Tejupilco, Mexico & Jiménez-Sánchez et al. 2000 \\
\hline Philonthus sp.2 & Pig & Campinas, Brazil & Souza \& Linhares 1997 \\
\hline Philonthus sp. 2 & Squid & Tlayacapan, Mexico & Márquez-Luna 2001 \\
\hline Philonthus sp.2 & Squid & Tejupilco, Mexico & Jiménez-Sánchez et al. 2000 \\
\hline Philonthus sp.2 & Pig & Curitiba, Brazil & Mise et al. 2007 \\
\hline Philonthus sp. 3 & Pig & Campinas, Brazil & Souza \& Linhares 1997 \\
\hline Philonthus sp. 3 & Squid & Tejupilco, Mexico & Jiménez-Sánchez et al. 2000 \\
\hline Philonthus sp.3 & Pig & Curitiba, Brazil & Mise et al. 2007 \\
\hline Philonthus sp.4 & Squid & Tejupilco, Mexico & Jiménez-Sánchez et al. 2000 \\
\hline Philonthus sp.4 & Pig & Curitiba, Brazil & Mise et al. 2007 \\
\hline Philonthus sp.5 & Squid & Tejupilco, Mexico & Jiménez-Sánchez et al. 2000 \\
\hline Philonthus sp.5 & Pig & Curitiba, Brazil & Mise et al. 2007 \\
\hline Philonthus sp.6 & Pig & Curitiba, Brazil & Mise et al. 2007 \\
\hline Philothalpus paederiformis Sharp & Squid & Tlayacapan and Tejupilco, Mexico & $\begin{array}{l}\text { Jiménez-Sánchez et al. 2000; Márquez- } \\
\text { Luna } 2001\end{array}$ \\
\hline Philothalpus subtilis Sharp & Squid & Tlayacapan and Tejupilco, Mexico & $\begin{array}{l}\text { Jiménez-Sánchez et al. 2000; Márquez- } \\
\text { Luna } 2001\end{array}$ \\
\hline Phloeonomus centralis Sharp & Squid & Tejupilco, Mexico & $\begin{array}{l}\text { Jiménez-Sánchez et al. 2000; Márquez- } \\
\text { Luna } 2001\end{array}$ \\
\hline Phloeonomus sp. & Squid & Tejupilco, Mexico & Jiménez-Sánchez et al. 2000 \\
\hline Phloeonomus sp. & Squid & Tlayacapan, Mexico & Márquez-Luna 2001 \\
\hline Platydracus biseriatus (Sharp) & Squid & Tlayacapan, Mexico & Márquez-Luna 2001 \\
\hline Platydracus castaneus (Nordmann) & Squid & Tejupilco, Mexico & Jiménez-Sánchez et al. 2000 \\
\hline Platydracus fervidus Sharp & Squid & Tejupilco, Mexico & Jiménez-Sánchez et al. 2000 \\
\hline Platydracus marcidus (Sharp) & Squid & Tlayacapan, Mexico & Márquez-Luna 2001 \\
\hline Platydracus mendicus (Sharp) & Squid & Tlayacapan and Tejupilco, Mexico & $\begin{array}{l}\text { Jiménez-Sánchez et al. 2000; Márquez- } \\
\text { Luna } 2001\end{array}$ \\
\hline Platydracus sp. & Pig & Curitiba, Brazil & Mise et al. 2007 \\
\hline Platydracus sp. 1 & Squid & Tlayacapan, Mexico & Márquez-Luna 2001 \\
\hline Platydracus sp. 1 & Squid & Tejupilco, Mexico & Jiménez-Sánchez et al. 2000 \\
\hline Platydracus sp. 2 & Squid & Tlayacapan, Mexico & Márquez-Luna 2001 \\
\hline Platydracus sp. 2 & Squid & Tejupilco, Mexico & Jiménez-Sánchez et al. 2000 \\
\hline Platydracus sp. 3 & Squid & Tlayacapan, Mexico & Márquez-Luna 2001 \\
\hline
\end{tabular}


Table I. Cont.

\begin{tabular}{|c|c|c|c|}
\hline Families/species & $\begin{array}{l}\text { Substratum/ } \\
\text { carcasses }\end{array}$ & Geographic distribution & References \\
\hline Platydracus sp. 3 & Squid & Tejupilco, Mexico & Jiménez-Sánchez et al. 2000 \\
\hline Proteinus sp. & Squid & Tlayacapan, Mexico & Márquez-Luna 2001 \\
\hline Pseudopsis sp. complexo "sulcata" & Squid & Tlayacapan, Mexico & Márquez-Luna 2001 \\
\hline Pseudopsis sp. & Pig & Medellín, Colombia & Wolff et al. 2001 \\
\hline Quedius sp. & Squid & Tlayacapan, Mexico & Márquez-Luna 2001 \\
\hline Quedius sp. & Pig & Curitiba, Brazil & Mise et al. 2007 \\
\hline Quedius sp.1 & Squid & Tejupilco, Mexico & Jiménez-Sánchez et al. 2000 \\
\hline Quedius sp.2 & Squid & Tejupilco, Mexico & Jiménez-Sánchez et al. 2000 \\
\hline Reichenbachia sp. & Squid & Tlayacapan, Mexico & Márquez-Luna 2001 \\
\hline Rugilus sp.1 & Squid & Tlayacapan, Mexico & Márquez-Luna 2001 \\
\hline Rugilus sp.2 & Squid & Tlayacapan, Mexico & Márquez-Luna 2001 \\
\hline Sepedophilussp. & Squid & Tejupilco, Mexico & Jiménez-Sánchez et al. 2000 \\
\hline Sepedophilus sp.1 & Squid & Tlayacapan, Mexico & Márquez-Luna 2001 \\
\hline Sepedophilus sp.2 & Squid & Tlayacapan, Mexico & Márquez-Luna 2001 \\
\hline Spedophilus sp.(sic) & Pig & Medellín, Colombia & Wolff et al. 2001 \\
\hline Stamnoderus sp. & Squid & Tejupilco, Mexico & Jiménez-Sánchez et al. 2000 \\
\hline Stamnoderus sp.1 & Squid & Tlayacapan, Mexico & Márquez-Luna 2001 \\
\hline Stamnoderus sp. 2 & Squid & Tlayacapan, Mexico & Márquez-Luna 2001 \\
\hline Stenus aff. popocatepetlensis & Squid & Tlayacapan, Mexico & Márquez-Luna 2001 \\
\hline Stenus sp.(?) & Pig & Medellín, Colombia & Wolff et al. 2001 \\
\hline Styngetus adrianae Navarrete & Squid & Tlayacapan and Tejupilco, Mexico & $\begin{array}{l}\text { Jiménez-Sánchez et al. 2000; Márquez- } \\
\text { Luna } 2001\end{array}$ \\
\hline Tachinomorphus grandis (Solsky) & Squid & Tlayacapan, Mexico & Márquez-Luna 2001 \\
\hline Thinocharis sp. & Squid & Tlayacapan and Tejupilco, Mexico & $\begin{array}{l}\text { Jiménez-Sánchez et al. 2000; Márquez- } \\
\text { Luna } 2001\end{array}$ \\
\hline Thoracophorus sp. & Pig & Curitiba, Brazil & Mise et al. 2007 \\
\hline Thyreocephalus puncticeps Sharp & Squid & Tlayacapan and Tejupilco, Mexico & $\begin{array}{l}\text { Jiménez-Sánchez et al. 2000; Márquez- } \\
\text { Luna } 2001\end{array}$ \\
\hline Toxidium punctatum Matthews & Squid & Tlayacapan, Mexico & Márquez-Luna 2001 \\
\hline Xanthopygus sp. & Pig & Campinas, Brazil & Souza \& Linhares 1997 \\
\hline Xenopygus analis (Erichson) & Squid & Tejupilco, Mexico & Jiménez-Sánchez et al. 2000 \\
\hline Philonthina sp. & Pig & Curitiba, Brazil & Mise et al. 2007 \\
\hline Trimiina sp. & Squid & Tlayacapan, Mexico & Márquez-Luna 2001 \\
\hline Oxypodini sp. & Pig & Curitiba, Brazil & Mise et al. 2007 \\
\hline Staphylininae sp. & Pig & Curitiba, Brazil & Mise et al. 2007 \\
\hline Staphylinidae sp. & Dog & San José, Costa Rica & Jirón \& Cartín 1981 \\
\hline Staphylinidae sp. & Rat & Campinas, Brazil & Monteiro-Filho \& Penereiro 1987 \\
\hline Staphylinidae sp. 1 & Pig & Recife, Brazil & Cruz \& Vasconcelos 2006 \\
\hline Staphylinidae sp. 2 & Pig & Recife, Brazil & Cruz \& Vasconcelos 2006 \\
\hline Staphylinidae sp. & Rat & Altos de Pipe (Caracas), Venezuela & Velásquez, 2007 \\
\hline Staphylinidae spp. & Pig & $\begin{array}{l}\text { Campinas, Brazil; Medellín, } \\
\text { Colombia }\end{array}$ & $\begin{array}{l}\text { Carvalho et al. 2000; Wolf et al. 2001; } \\
\text { Carvalho et al. } 2004\end{array}$ \\
\hline \multicolumn{4}{|l|}{ Tenebrionidae } \\
\hline Lagria vilosa (Fabricius) & Pig & Curitiba, Brazil & Mise et al. 2007 \\
\hline Prostenus periscelis (Perty) & general & São Paulo, Brazil & Luederwaldt 1911 \\
\hline Xystropus femoratus (Germar) & general & São Paulo, Brazil & Luederwaldt 1911 \\
\hline Allecullidae sp. (=Tenebrionidae) & Pig & Recife, Brazil & Cruz \& Vasconcelos 2006 \\
\hline Tenebrionidae sp. 1 & Pig & Curitiba, Brazil & Mise et al. 2007 \\
\hline Tenebrionidae sp. 2 & Pig & Curitiba, Brazil & Mise et al. 2007 \\
\hline Tenebrionidae sp. 3 & Pig & Curitiba, Brazil & Mise et al. 2007 \\
\hline Tenebrionidae sp. 4 & Pig & Curitiba, Brazil & Mise et al. 2007 \\
\hline \multicolumn{4}{|l|}{ Trogidae } \\
\hline Omorgus sp. & Pig & Curitiba, Brazil & Mise et al. 2007 \\
\hline Polynoncus sp. & Pig & Curitiba, Brazil & Mise et al. 2007 \\
\hline $\begin{array}{l}\text { Trox gemmingeri (Harold) } \\
(=\text { Polynoncus })\end{array}$ & general & São Paulo, Brazil & Luederwaldt 1911 \\
\hline Trox pilularius (Germar) (=Polynoncus) & general & São Paulo, Brazil & Luederwaldt 1911 \\
\hline Trox suberosus (Fabricius) & general & São Paulo, Brazil & Luederwaldt 1911 \\
\hline Trogidae $\mathrm{sp}$. & Rat & Curitiba, Brazil & Moura et al. 1997 \\
\hline
\end{tabular}


Neotropical region. They are mainly predators of soft body insects larvae and eggs, particularly those of Cyclorraphan Diptera. Most occur in carrion, dung, decomposing plant materials, such as fungi, and tree wounds. Some live under loose bark or in galleries of wood-boring insects, where they prey on other organisms. The greatly flattened species live under bark of dead or dying trees. Cylindrical species occur in tunnels of bark beetles and other wood-boring insects. Most species are neither flattened nor cylindrical and are abundant in the early stages of decay of carcasses.

Hydrophilidae. Water scavenger beetles. A family with about 140 genera and 2,025 species, with 42 genera and 3,064 species in the Neotropical region. Larvae and adults of many are aquatic or semiaquatic, but some live in fresh mammal dung, humus-rich soil, or decaying leaves. Adults are mainly scavengers but the larvae are usually predacious. The terrestrial species occur in dung.

Leiodidae. Round fungus beetles. A family with about 334 genera and 4,240 species, with 38 genera and 279 species in the Neotropical region. Most species occur in carrion but some are found in fungi, some feed on slime molds, and others occur in ant nests.

Nitidulidae. Sap beetles. A family with about 160 genera and 3,000 species, with 79 genera and 770 species in the Neotropical region. They have a varied biology. Many are phytophagous, including pollen feeders, seeds, tree sap, others feed on dead or decaying plants, rotten fruits, leaf litter and a few are carrion feeders. Some are predators of scale insects.

Scarabaeidae. Scarab beetles. A family with about 2,000 genera and 25,000 species, with 362 genera and 4,706 species in the Neotropical region. Adults and larvae may be detritivorous, saprophagous, herbivorous, necrophagous or coprophagous. Larvae of most injurious species live in soil, feeding on roots, others may feed in rotten wood, dry carrion or skins.

Silphidae. Carrion beetles. A family with about 14 genera and 175 species, with 9 genera and 82 species in the Neotropical region. Most common on carrion but sometimes found on decaying vegetation or living plants. They feed on maggots and also are associated with vertebrate carcasses.

Staphylinidae. Rove beetles. A family with about 659 genera and 48,000 species, with 652 genera and 8,124 species in the Neotropical region. Adults are found in a wide range of habitats, under stones and other objects on ground, along shores of streams and lakes. Some live along ocean shores, on carrion, in manure, on fungi, on flowers in ant and termite nests, under bark, in soil or soil litter and in caves. They are among the most proficient fliers of all beetles. Many species run with the tip of abdomen raised. Larvae usually occur in the same habitat as adults. Most species are saprophagous feeding on dead or decaying plant or animal materials, such as carrion, dung, dead $\operatorname{logs}$, etc. Most species are predacious and a few are parasites of other insects.

Tenebrionidae. Darkling beetles. A family with about 1,700 genera and 18,000 species, with 478 genera and 4,624 species in the Neotropical region. Adults and larvae live in a variety of terrestrial habitats. Some lives in rooting wood, on plant materials, under logs and stones, in termite and ant nests, in houses, in fungi and in debris. They feed on material of plant origin including decaying plant litter, dead wood, fungal fruiting bodies. Some feed on dead animal material and a few are predators. A few species are pests of stored products and the root-feeding larvae can also be agricultural pests specially of young plants and during dry conditions.

Trogidae. Hide beetles. A family with about 5 genera and 300 species, with 3 genera and 48 species in the Neotropical region. Adults and larvae are among the last inhabitants of fish, amphibians, reptiles, birds and mammals carcasses. The species of the genus Trox occur in nests of birds and burrows of mammals, especially those with masses of fur or feathers. When disturbed these beetles draw in their legs and lie motionless resembling dirt and rubbish and are often overlooked.

Acknowledgments. The authors thank to doctors José Roberto Pujol-Luz, Universidade de Brasília; José Albertino Rafael and Ruth Leila Ferreira Keppler, Instituto Nacional de Pesquisas da Amazônia; Marta Wolff, Instituto de Biología de la Universidad de Antioquia; Janyra Oliveira-Costa, Universidade Castelo Branco and Rodrigo Krüger, Universidade Federal de Viçosa, for loan of the material. To Nicolas Dégallier; Carla de Lima Bicho (Histeridae); Pedro Gnaspini Netto (Leiodidae); Edilson Caron (Staphylinidae) and Paschoal Coelho Grossi (Scarabaeidae) for aid in identifying the material.

\section{REFERENCES}

Ahmed, A. R.; R. Moy; A. R. Barr \& Z. Prize. 1981. Carpet beetle dermatitis. Journal of the American Academy of Dermatology 5: 428-432.

Ashman, F. 1963. Factors affecting the abundance of the copra beetle Necrobia rufipes (De Geer) (Coleoptera, Cleridae). Bulletin of Entomological Research 53: 671-680.

Booth, R. G.; M. L. Cox \& R. B. Madge. 1990. 3. Coleoptera IIE. Guides to insects of importance to man. Cambridge, Cambridge University Press, vi+384 pp.

Borror, D. J.; C. A. Triplehorn \& N. F. Johnson. 1992. An Introduction to the study of insects $\left(6^{a}\right.$ ed.). Fort Worth, Saunders College Publishing, Harcourt Brace College Publishers, xiv+875 pp.

Carvalho, L. M. L.; P. J. Thyssen; A. X. Linhares \& F. A. B. Palhares. 2000. A checklist of Arthropods associated with pig carrion and human corpses in southeastern Brazil. Memórias do Instituto Oswaldo Cruz 95: 135-138.

Carvalho, L. M. L.; P. J. Thyssen; M. L. Goff \& A. X. Linhares. 2004. Observations on the succession patterns of necrophagous insects on a pig carcass in an urban area of southeastern Brazil. Aggrawal's Internet Journal of Forensic Medicine and Toxicology 5: $33-39$.

Centeno, N.; M. Maldonado \& A. Oliva. 2002. Seasonal patterns of 
arthropods occurring on sheltered and unsheltered pig carcasses in Buenos Aires province (Argentina). Forensic Science International 126: $63-70$.

Costa, C.; S. Ide \& C. E. Simonka. 2006. Insetos imaturos. Metamorfose e identificação. Ribeirão Preto, Holos Editora, 249 p.

Costa, C. 2000. Estado de conocimiento de los Coleoptera neotropicales, pp. 99-114. In: F., Martín-Piera, J. J. Morrone, \& A. Melic (eds). PrIBES. Proyecto Iberoamericano de Biogeografía y Entomología Sistemática. Zaragoza. Vol. 1. Villa de Leyva, Colômbia, Sociedad Entomológica Aragonesa, 326p.

Cruz, T. M. \& S. D. Vasconcelos. 2006. Entomofauna de solo associada à decomposição de carcaça suína em um fragmento de Mata Atlântica de Pernambuco, Brasil. Biociências 14: 193-201.

Goff, M. L. 1991. Comparison of insect species associated with decomposing remains recovered inside dwellings and outdoors on the island of Oahu, Hawaii. Journal of Forensic Sciences 36: $748-753$.

Gredilha, R.; P. R. Saavedra; L. G. Guerim; A. F. Lima \& N. M. SerraFreire. 2005. Ocorrência de Oryzaephilus surinamensis Linnaeus, 1758 (Coleoptera: Cucujidae) e Necrobia rufipes DeGeer, 1775 (Coleoptera: Cleridae) infestando rações de animais domésticos. Entomologia Y Vectores, 12: 95-103.

Iannacone, J. 2003. Artropofauna de importancia forense en un cadáver de cerdo en el Callao, Perú. Revista Brasileira de Zoologia 20: 85-90.

Jimenez-Sanchez, E.; J. L. Navarrete-Heredia \& J. R. Padilla-Ramirez. 2000. Estafilinidos (Coleoptera: Staphylinidae) necrofilos de la sierra de Nanchititla, Estado de Mexico, Mexico. Folia de Entomologia Mexicana 108: 53-78.

Jirón, L. F. \& V. M. Cartín. 1981. Insect succession in the decomposition of a mammal in Costa Rica. Journal of New York Entomological Society 89: $158-165$.

Kingsolver, J. M. 1991. Dermestid Beetles (Dermestidae, Coleoptera), p. 115-135. In: J.R. Gorham (ed.) Insect and Mite Pests in Food. Vol. 1. Washington, D.C., U.S. Government Printing Office, vii+310 p.

Kumar, P.; C. A. Jayaprakas; B. D. Singh \& K. Sengupta. 1988. Studies on the biology of Dermestes ater (Coleoptera: Dermestidae) - a pest of silkworm pupae and adults. Current Science 57: 1253.

Luederwaldt, G. 1911. Os insectos necrophagos paulistas. Revista do Museu Paulista 8: 414-433.

Márquez-Luna, J. 2001. Especies necrofilas de Staphylinidae (Insecta: Coleoptera) del municipio de Tlayacapan, Morelos, Mexico. Folia Entomologica Mexicana 40: 93-131.

Mise, K. M.; L. M. Almeida \& M. O.Moura. 2007. Levantamento da fauna de Coleoptera que habita a carcaça de Sus scrofa L., em Curitiba, Paraná. Revista Brasileira de Entomologia 51: 358 368.

Moura, M. O.; C. J. B. de Carvalho \& E. L. A. Monteiro-Filho. 1997. A preliminary analysis of insects of medico-legal importance in Curitiba, state of Paraná. Memórias do Instituto Oswaldo Cruz 92: 269-274.

Monteiro-Filho, E. L. A. \& J. L. Penereiro. 1987. Estudo de decomposição e sucessão sobre uma carcaça animal numa área do estado de São Paulo, Brasil. Revista Brasileira de Biologia 47: 289-295.

Newton, A. F.; C. Gutiérrez Chacón \& D. S. Chandler. 2005. Checklist of the Staphylinidae (Coleoptera) of Colombia. Biota Colombiana 6: $1-72$.

Pessôa, S. B. \& F. Lane. 1941. Coleópteros Necrófagos de Interêsse Médico-Legal. Ensaio monográfico sobre a família Scarabaeidae de S. Paulo e regiões visinhas. Revista do Museu Paulista 25: 389504.

Scampini, E.; A. Cichino \& N. Centeno. 2002. Especies de Carabidae (Coleoptera) asociadas a cadavers de cerdo (Sus scrofa L.) en Santa Catalina (Buenos Aires, Argentina). Revista de la Sociedad Entomologica Argentina 61: $85-88$.

Smith, K. G. V. 1986. A manual of forensic entomology. Ithaca, NY, Cornell University Press, 205 p.

Souza, A. M. \& A. X. Linhares. 1997. Diptera and Coleoptera of potential forensic importance in southeastern Brazil: relative abundance and seasonality. Medical and Veterinary Entomology 11: $8-12$.

Turner Jr., E.C. 1986. Structural and litter Pests. Poultry Science 65: 644-648.

Veer, V.; B. K. Negi \& K. M. Rao. 1996. Dermestid beetles and some other insect pests associated with stored silkworm cocoons in India, including a world list of dermestid species found attacking this commodity. Journal of Stored Products Research 32: 69-89.

Velásquez, Y. 2007. A checklist of arthropods associated with rat carrion in a montane locality of northern Venezuela. Forensic Science International 174: 68-70.

White, R. E. 1983. A field guide to the beetles of North America. The Peterson field guide series 29. Boston, Houghton Mifflin Company, 368 p.

Wildey, K. B. \& C. Wayman. 1979. The hide beetle (Dermestes maculatus) as a deep pit pest in poultry houses. ADAS Quarterly Review 34: 187-193.

Wolff, M.; A. Uribe; A. Ortiz \& P. Duque. 2001. A preliminary study of forensic entomology in Medellín, Colombia. Forensic Science International 120: $53-59$.

Received 31/10/2008; accepted 20/03/2009 